\title{
1 A modal approach to piano soundboard vibroacoustic behavior
}

2 Authors: Benjamin Trévisan, Kerem Ege and Bernard Laulagnet

3 Affiliation: Univ Lyon, INSA-Lyon, LVA EA677, F-69621, Villeurbanne, France

4 Corresponding authors mail address: benjamin.trevisan@insa-lyon.fr

5 Submission or revision date: 2 Jan. 17

\section{Abstract}

7 This paper presents an analytical method for modeling the vibro-acoustic behavior of

8 ribbed non-rectangular orthotropic clamped plates. To do this, the non-rectangular plate

9 is embedded in an extended rectangular simply supported plate on which a spring

10 distribution is added, blocking the extended part of the surface, and allowing the

11 description of any inner surface shapes. The acoustical radiation of the embedded plate

12 is ensured using the radiation impedances of the extended rectangular simply

13 supported plate. This method is applied to an upright piano soundboard: a

14 non-rectangular orthotropic plate ribbed in both directions by several straight stiffeners.

15 A modal decomposition is adopted on the basis of the rectangular extended simply

16 supported plate modes, making it possible to calculate the modes of a piano

17 soundboard in the frequency range $[0 ; 3000] \mathrm{Hz}$, showing the different associated mode

18 families. Likewise, the acoustical radiation is calculated using the radiation impedances

19 of a simply supported baffled plate, demonstrating the influence of the string coupling

20 point positions on the acoustic radiated power. The paper ends with the introduction of

21 indicators taking into account spatial and spectral variations of the excitation depending

22 on the notes, which add to the accuracy of the study from the musical standpoint. A 
1 parametrical study, which includes several variations of soundboard design, highlights

2 the complexity of rendering high-pitched notes homogeneous. 


\section{I. INTRODUCTION}

2 Traditionally designed using empirical approaches, musical instruments are now being

3 studied regarding the perceptive and subjective aspects of the sounds they produce.

4 Indeed, many parameters influence their timbres, ranging from the wood used [1] to

5 specifications linked to their design, which greatly determines their vibratory behavior.

6 In the case of the piano, the soundboard plays an essential role in the functioning of the

7 instrument. Indeed, string sections are too thin to radiate on their own. Thus their

8 vibrations are transmitted to the soundboard through bridges that serve as effective

9 acoustic radiators. The geometry of a soundboard is complex and composed of a

10 non-rectangular plate, traditionally made of spruce (orthotropic material), ribbed on one

11 side by several beams placed perpendicular to the wood fibers, and by one or two

12 bridges almost parallel to the fibers, on the opposite side. Fig. 1 shows an example of

13 such a structure.

14 The vibro-acoustic broadband behavior of this structure has been studied by several

15 authors including Fletcher [2], Weinrech [3], and Kindel [4], then by Suzuki [5], [6],

16 Conklin [7]-[9], Giordano [10]-[12], Dérogis [13], Berthaut [14], Bensa [15], Stulov [16],

17 and more recently by Ege and Boutillon [17], [18], Chaigne [19], Chabassier [20],

18 Rigaud [21], and Etcheniquel [22]. It is now the subject of software development for

19 piano sound synthesis [23].

20 Many other works already exist on the subject of ribbed structures and not only in the

21 area of musical instruments. Indeed, nowadays simple structures like beams and

22 isotropic plates are described well [24]-[33], but the design and modeling of ribbed 
1 structures nonetheless remains a major topic of research. Most of these studies have

2 focused on periodically ribbed isotropic flat plates [34]-[44], curved panels [45],

3 stiffened cylindrical shells [46]-[53], and more recently laminated composite panels [54],

$4 \quad[55]$ and sandwich structures [56]-[59].

5 Problems linked to the piano soundboard have been studied from the angle of purely

6 musical issues. These included the quest for a good compromise between "sustain /

7 radiated power" and poor color in high frequencies (i.e poor spectrum of last notes),

8 both of which appear to be the main difficulties confronting piano makers.

9 Although modelling flat ribbed structures and their baffled radiation has long been an

10 area of research, taking into account non-rectangular geometry, non-special orthotropy

11 (i.e. the axes of orthotropy are not parallel to the boundary if rectangular) and attached

12 bars in orthogonal directions simultaneously is a real challenge.

13 With a view to solving these issues, modelling is performed using a variational approach

14 in which the judicious use of simply supported rectangular extended plate modes and

15 radiation impedances is introduced. The aim of this model of an upright piano

16 soundboard, which avoids any object discretization, is to provide an interesting

17 alternative to a complete numerical method such as the FEM-BEM method (with long

18 computing time), which could obviously be used.

19 Consequently, this model facilitates parametrical studies and, for example, highlights

20 the influence of ribs and bridges (numbers, width, length, height) on the acoustical

21 radiation of the piano soundboard and, more generally, on orthotropic ribbed panels. 
1 This article is the continuation of works presented in [60]. The first part of the article

2 presents how the non-rectangular edges and orientation of the materials composing the

3 piano soundboard are formulated by the addition of springs. Then, in the second part,

4 the model of the soundboard of a Pleyel P131 is described. It comprises the addition of

5 ten ribs, two bridges and two "cut-off" corner beams. The modal aspects are presented

6 in the frequency range $[0 ; 3000] \mathrm{Hz}$. In particular we discuss the different families of

7 modes due to stiffening. Then, several numerical results including mobility and acoustic

8 radiated power are presented for an excitation moving along the main bridge at the

9 position of the string couplings. We show how the vibro-acoustical behavior of the

10 soundboard depends on the positions of the strings, and how the discrete frequencies

11 of the notes (the sources) make analysis difficult.

12 Drawing its inspiration from [61], [62], a parametrical study shows the complexity of

13 obtaining homogeneous high-pitched notes and how it is related to the soundboard

14 design (main bridge discontinuity, ribs in the high frequency domain, etc.). This is done

15 by introducing a new indicator applied to the mobility and the acoustic radiated power,

16 taking into account the discrete spacing aspects (discontinuous excitation points) and

17 the discrete frequencies of the keys at the same.

\section{II. ORTHOTROPY WITH UNSPECIFIED ANGLE}

19 In traditional piano soundboard design, the direction of the wood fibers is not parallel to

20 the edges: we define this as the angle of orthotropy $\theta$. See Fig. 1. In this part we focus

21 on a method of modeling the orientation of the fibers and describing the non-rectangular

22 contours. We demonstrate the originality of this method which allows us to describe, 
1 from the analytical standpoint, any shapes embedded within a rectangular shape, and to

2 take into account non-specified orthotropy, i.e. when the main axes of orthotropy are not

3 parallel to the soundboard shapes.

4

5

6

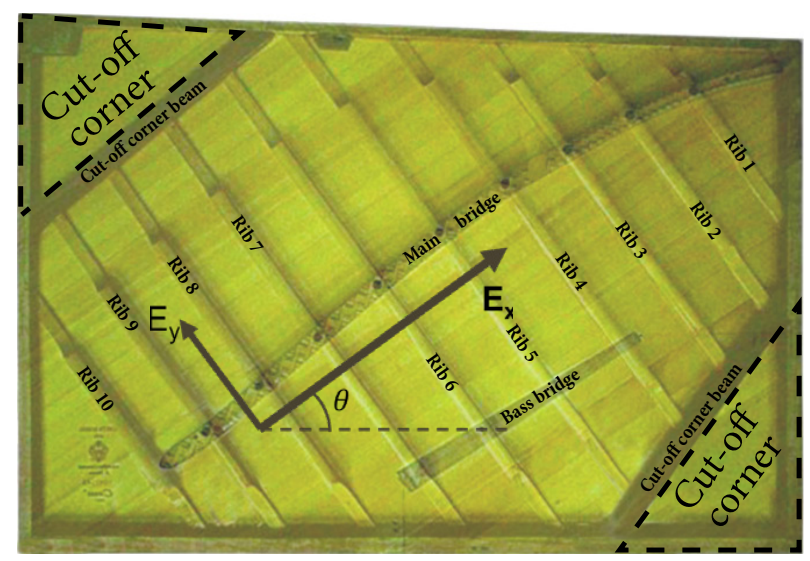

Fig. 1 - (Color online) Upright piano soundboard of Pleyel P131 with $E_{x}$ and $E_{y}$ being the strong and the weak Young's moduli of the wood. View from front side (bridge side) with ribs and cut-off corner beams added in transparency.

\section{II.A. THEORETICAL FORMULATION}

In order to describe both the non-rectangular contours and the unspecified angle of orthotropy, the following model uses the simple basis of a special rectangular orthotropic plate, i.e. the Young's moduli are parallel to the plate edges. See Fig. 2 on the left. By adding several springs (dark points) to block the transverse displacement, we describe the non-rectangular contours and the unspecified angle, as shown in Fig. 2 on the right. The boundary conditions will tend to clamped conditions. 

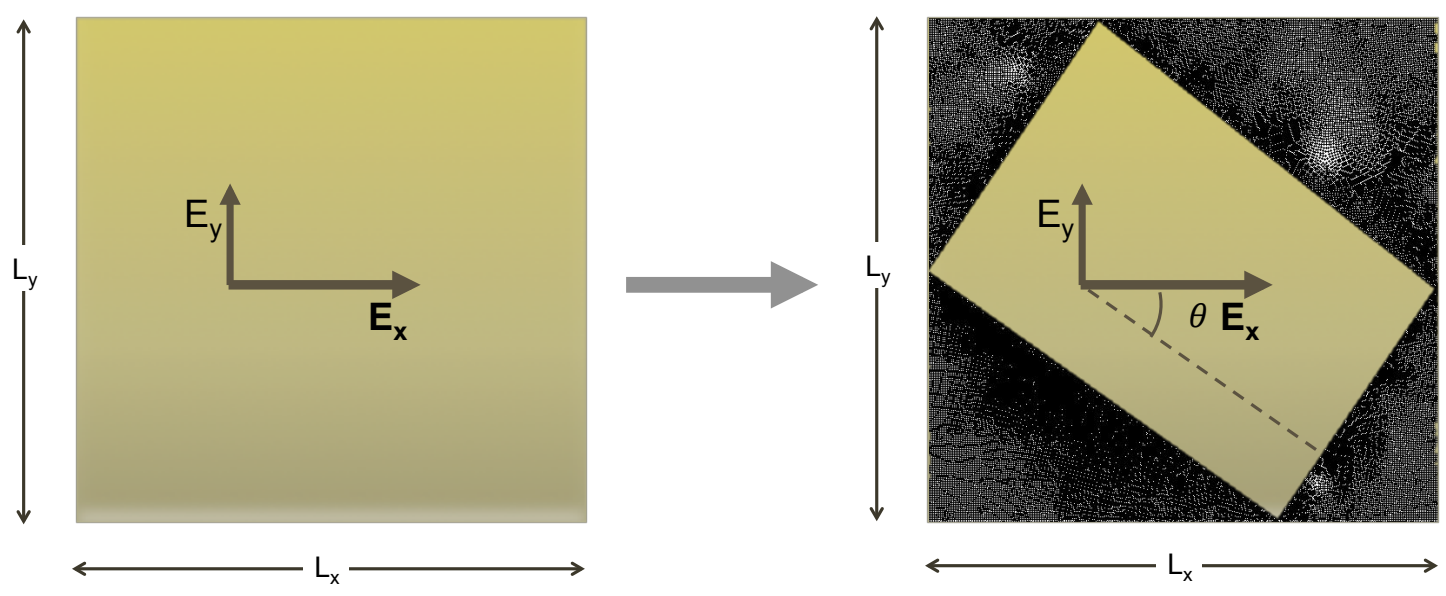

Fig. 2 - (Color online) The addition of springs on a rectangular simply supported plate. On the left: the initial plate with specific orthotropy; on the right: the addition of springs. The non-rectangular contours and the non-specified angle of orthotropy are well described.

Thus it is possible to describe any edges the user wishes (not limited to only soundboards) as suggested in Fig. 3. Obviously, it is possible to model a grand piano but we chose to study a Pleyel P131 upright soundboard available at the lab (kindly given by ITEMM, European Technological Institution for Music Professions).

a)

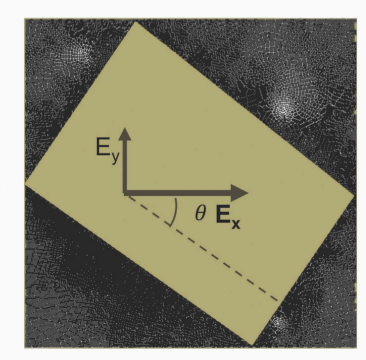

b)

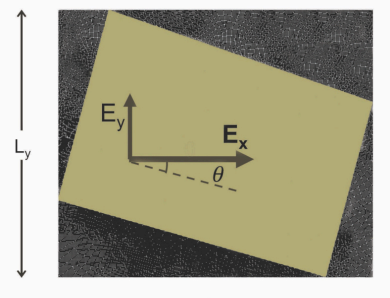

c)

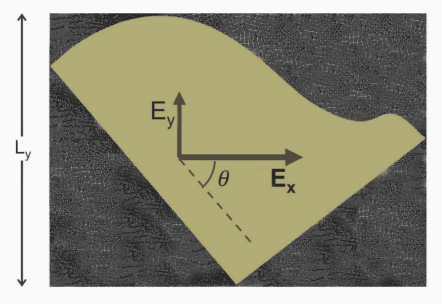

9

Fig. 3 - (Color online) Examples of possible geometries with different orthotropy angles and edges. 
are considered). An additional external force will be introduced in II.A.3 for the forced response calculation.

The starting point of our modeling is a simply supported rectangular plate with specific orthotropy. It follows the thin plate Love-Kirchhoff hypothesis for which the Hamiltonian can be easily found in the literature [25]. Additional information on the kinetic hypothesis can be found in a previous article [60].

We consider several springs coupled to the plate at the coordinates $\left(x_{s}, y_{s}\right)$. We give the following Hamiltonian for a plate with a number of springs $\mathrm{N}_{\mathrm{s}}$ :

$$
\begin{aligned}
H_{o p}=\int_{t_{0}}^{t_{1}} \frac{1}{2} \int_{x=0}^{L_{x}} \int_{y=0}^{L_{y}} \rho h w^{2}-\left(D_{1} w_{, x x}^{2}+D_{3} w_{, y y}^{2}+D_{2} w_{, x x} w_{, y y}+D_{4} w_{, x y}^{2}\right) d x d y d t \\
+\sum_{s=1}^{N_{s}} \int_{t_{0}}^{t_{1}}-\frac{1}{2} \int_{x=0}^{L_{x}} \int_{y=0}^{L_{y}} k w^{2}(x, y) \delta\left(x-x_{s}\right) \delta\left(y-y_{s}\right) d x d y d t
\end{aligned}
$$

with $w$ being the plate transverse displacement; $k$ the spring rigidity; $\left[t_{0} ; t_{1}\right]$ an arbitrary time interval; $L_{x}$ and $L_{y}$ the plate dimensions; $\rho$ the plate mass density; $h$ the plate thickness; $D_{1}=\frac{E_{x} h^{3}}{12\left(1-v_{x y} v_{y x}\right)}, D_{3}=\frac{E_{y} h^{3}}{12\left(1-v_{x y} v_{y x}\right)}, D_{2}=\frac{v_{y x} E_{x} h^{3}}{6\left(1-v_{x y} v_{y x}\right)}$ and $D_{4}=\frac{G_{x y} h^{3}}{3}$ the plate dynamic stiffness; $v_{x y}$ and $v_{y x}=v_{x y} E_{y} / E_{x}$ its Poisson coefficients; $E_{x}$ and $E_{y}$ the two Young moduli of the plate. The first line of the equation is related to the plate and the second to the springs where the Dirac distributions render them localized at the coordinates $\left(x_{s}, y_{s}\right)$. Table 1 gives the properties of the extended rectangular orthotropic spruce plate (Fig. 2). 


\section{II.A.2. MODAL DECOMPOSITION}

As mentioned previously, the starting point of our model is a rectangular simply supported plate. This basis, currently used in the area of vibrations, is particularly adapted for an analytical approach and has been studied several times [26], [46], [63]. Of course, this basis is not the real basis of the embedded plate but only a convenient basis for our model, as will be shown in the following. Using modal decomposition, the transverse displacement is written as a linear combination of simply supported plate modes weighted by modal amplitudes $a_{m n}(t)$ :

$$
w(x, y, t)=\sum_{m=1}^{M} \sum_{n=1}^{N} a_{m n}(t) \phi_{m n}(x, y) \forall x \in\left[0 ; L_{x}\right] \text { and } y \in\left[0 ; L_{y}\right]
$$

where $\phi_{m n}(x, y)=\sin \left(m \pi / L_{x} x\right) \sin \left(n \pi / L_{y} y\right)$.

By injecting the modal decomposition (2) into Eq. (1) and using the orthogonal properties of eigenvectors, it is possible to analytically calculate the surface integral in the Hamiltonian. Thus the functional depends on the two variables $\left[a_{m n}(t), \dot{a}_{m n}(t)\right]$ and no longer on the transverse displacement $w(x, y, t)$ and its space and temporal derivatives. Thus we have:

$$
H_{o p}\left(a_{m n}(t), \dot{a}_{m n}(t)\right)=\int_{t_{0}}^{t_{1}} \mathcal{L}\left(a_{m n}(t), \dot{a}_{m n}(t) d t\right.
$$
where $\mathcal{L}\left(a_{m n}(t), a_{m n}(t)\right)$ is termed the Lagrangian of the system.

16 By applying the differential form of the principle of least action and using 17 Euler-Lagrange equations, the action of the system is minimized, leading to the 18 following homogeneous linear system: 


$$
\left\{\overline{\bar{M}}^{\text {plate }}+\overline{\bar{M}}^{\text {springs }}\right\} \ddot{\vec{a}}+\left\{\overline{\bar{K}}^{\text {plate }}+\overline{\bar{K}}^{\text {springs }}\right\} \vec{a}=\overrightarrow{0}
$$

where $\overline{\bar{M}}$ and $\bar{K}$ represent mass and rigidity matrices. These matrices are constituted by the sum of diagonal matrices coming from the plate and full and symmetrical matrices from the springs.

Solving the corresponding eigenvalue problem Eq. (4) allows calculating the modes of the embedded plate: eigen-frequencies and modal shapes, the matrix of eigenvectors and thus the transfer matrix $\overline{\bar{T}}$ between the initial simply supported basis and the system basis, which is the orthonormed basis of the eigenvectors [60]. We give the relation between the two bases with $\vec{a}$ and $\vec{b}$ being the vectors of modal amplitudes in the initial basis and in the new basis, respectively:

$$
\vec{a}=\overline{\bar{T}} \vec{b}
$$

Consequently, the real modal shapes of the embedded plate are built as a linear combination of the simply supported shapes of the extended plate:

$$
\Phi^{(i)}(x, y)=\sum_{m=1}^{M} \sum_{n=1}^{N} a_{m n}^{(i)} \phi_{m n}(x, y) \forall x \in\left[0 ; L_{x}\right] \text { and } y \in\left[0 ; L_{y}\right]
$$

Where $(i)$ indicates the column number in the matrix of eigenvectors.

Note that the size of these matrices, which is governed by the truncation on the orders $\mathrm{M}$ and $\mathrm{N}$ in the finite modal decomposition Eq. (2), determines the precision of the results.

\section{II.A.3. FORCED RESPONSE}

We assume that the fluid loading is negligible, so the light fluid assumption is made (for a full fluid loading coupling, see [26]). Here, we consider a sinusoidal 
and harmonic external force applied at point $\left(x_{e}, y_{e}\right)$ with an amplitude of $1 \mathrm{~N}$. Following the same approach as before, we determine the vector of generalized force $\vec{F}_{g e n}$ (second member of Eq. (4)) for which the components are defined by:

$$
F_{p q}=\phi_{p q}\left(x_{e}, y_{e}\right)
$$

To determine the forced response of the system we choose to express the 5 problem in the system basis. Using Eq. (5), the generalized matrix problem Eq. (4) 6 with the second member becomes diagonal:

$$
-\omega^{2} \overline{\bar{M}}_{o p} \vec{b}(\omega)+j \omega \overline{\bar{C}} \vec{b}(\omega)+\overline{\bar{K}}_{o p} \vec{b}(\omega)=\overline{\bar{T}}^{t} \vec{F}_{g e n}
$$

$$
\text { With } \overline{\bar{M}}_{o p}=\overline{\bar{T}}^{t}\left\{\overline{\bar{M}}^{\text {plate }}+\overline{\bar{M}}^{\text {springs }}\right\} \overline{\bar{T}} \text { and } \overline{\bar{K}}_{o p}=\overline{\bar{T}}^{t}\left\{\overline{\bar{K}}^{\text {plate }}+\overline{\bar{K}}^{\text {springs }}\right\} \overline{\bar{T}} \text {. }
$$

$8 \quad$ The modal damping matrix $\overline{\bar{C}}$ is also introduced. The structure is considered as a 9 weakly dissipative system for which the equations of generalized displacements 10 are uncoupled [64]-[66] so the matrix $\overline{\bar{C}}$ is also diagonal and defined by:

$$
\overline{\bar{C}}=\left(\begin{array}{lll}
\ddots & & \\
& \eta_{i} m_{i} \omega_{i} & \\
& & \ddots
\end{array}\right)
$$

11 With $m_{i}, \omega_{i}$ and $\eta_{i}$ being the modal mass, angular frequency and damping loss 12 factor of the $\mathrm{i}^{\text {th }}$ system mode.

13 In the case of a piano soundboard, the modal loss factor was evaluated between $141 \%$ and $3 \%$, depending on the modes in our frequency range of interest [67]. In the 15 following, the modal loss factors of all the soundboard modes are fixed at $2 \%$. 


\section{II.B. SPRING CALIBRATION}

2 Minimal spring rigidity and spacing are necessary to block transverse displacement.

3 For the frequency range of interest $[0 ; 3000] \mathrm{Hz}$, the spacing between springs 4 depends on the smallest wavelength in the plate. The latter is in the direction of the 5 weak Young's modulus [67]:

$$
\lambda=\sqrt{\frac{2 \pi}{f}}\left(\frac{D_{3}}{\rho h}\right)^{1 / 4}
$$

6 A criterion of $\lambda / 10$ is chosen to overestimate the number of springs. In the present 7 case, it leads us to a minimal distance between the springs of around $7.3 \mathrm{~mm}$ (for $\mathrm{f}=$ $8 \quad 3 \mathrm{kHz})$.

9 Minimal spring rigidity is also important: the ratio between the rigidity of the spring 10 and that of the plate must be sufficiently high. When the rigidity is insufficient, 11 vibrations appear at high frequencies in the "blocked" area (where the springs are 12 localized).

13 We define the ratio between the average quadratic velocity in the free area and the 14 whole plate as a criterion in order to validate sufficient spring rigidity:

$$
L_{v \text { ratio }}=10 \log \left(\frac{\int_{S_{\text {tot }}}|v(x, y)|^{2} d S}{\int_{S_{\text {int }}}|v(x, y)|^{2} d S}\right)
$$

15 For a sufficient value of spring rigidity the velocity on the blocked area will tend to 16 zero and thus this ratio will also tend to zero. 
Fig. 4-a presents this ratio for two spring rigidities. In both cases, the truncation order is fixed at $(P, Q)=(82,63)$ for which the convergence of the solution is guaranteed.

3 The excitation point is arbitrarily chosen near an edge.

a)

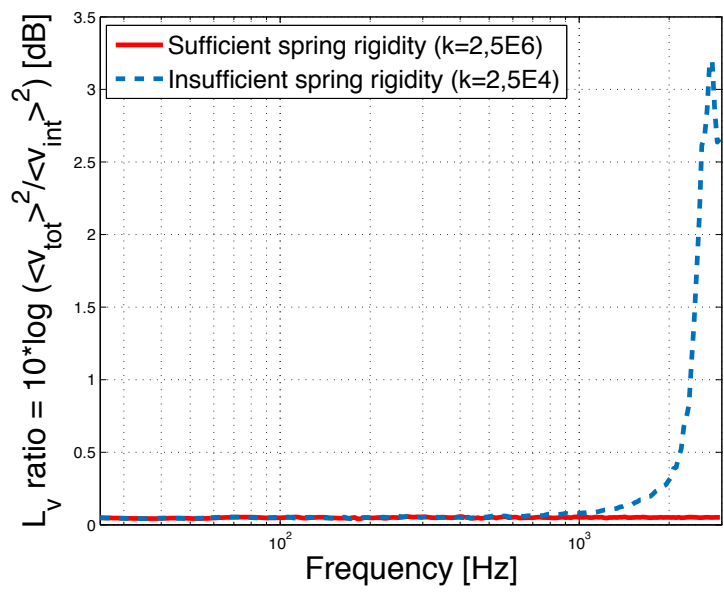

b)

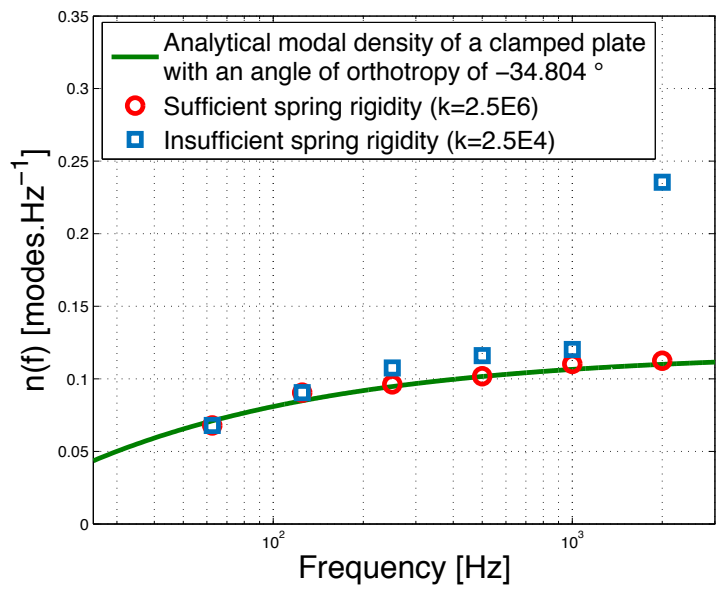

Fig. 4 - (Color online) Influence of spring rigidity.

6 a) Average quadratic velocity ratio for two spring rigidities. Solid line: sufficient rigidity; dashed line: insufficient rigidity. The excitation is placed at $\left(\mathrm{x}_{\mathrm{e}}, \mathrm{ye}_{\mathrm{e}}\right)=(1.26,0.76)$. markers: sufficient spring rigidity; square markers: insufficient spring rigidity; solid line: analytical expression.

10 When the springs are strong enough compared to the plate, the ratio is lower than

$$
0.06 \mathrm{~dB} \text { for the entire frequency range (solid line in Fig. 4-a). On the contrary, }
$$


1 insufficient springs imply a higher velocity ratio (dashed line in Fig. 4-a) from $1 \mathrm{kHz}$

2 with a peak around $3.2 \mathrm{~dB}$ at $2.5 \mathrm{kHz}$ in the present case. This lack of rigidity can

3 also be detected on the modal density (Fig. 4-b); see next paragraph.

$4 \quad$ Fig. 5 (on the left and center) presents modal shapes for these two cases. The modal

5 shapes are similar at low frequency. See top left and center of Fig. 5. Indeed, at low

6 frequency the ratios are equivalent. We can also note a small frequency difference.

7 From $1 \mathrm{kHz}$, three kinds of modes exist if the rigidity is insufficient: internal modes (in

8 the free area), external modes (in the density of springs) and hybrid modes (in the

9 two areas). The bottom left of Fig. 5 shows one of these undesired modes. On the

10 other hand, sufficient rigidity ensures only one kind of mode: the internal mode

11 (bottom center, Fig. 5).

12 As mentioned previously, the coexistence of these multi-type modes can also be

13 detected on the modal density. Fig. 4-b shows a comparison between the modal

14 density of the two cases of spring rigidity and the analytical formulation from [17],

15 [67]. This comparison shows good agreement of the modal density with the

16 theoretical plot (solid line in Fig. 4-b) when the rigidity is sufficient (circle markers in

17 Fig. 4-b) and tends toward the same value. On the contrary, when the spring rigidity

18 is insufficient, the presence of external modes implies a considerable increase of

19 modal density (square markers in Fig. 4-b). This effect is very interesting and

20 appears even if the order of truncation is not adapted. It can facilitate the detection of

21 external modes by solving only the eigenvalue problem. 

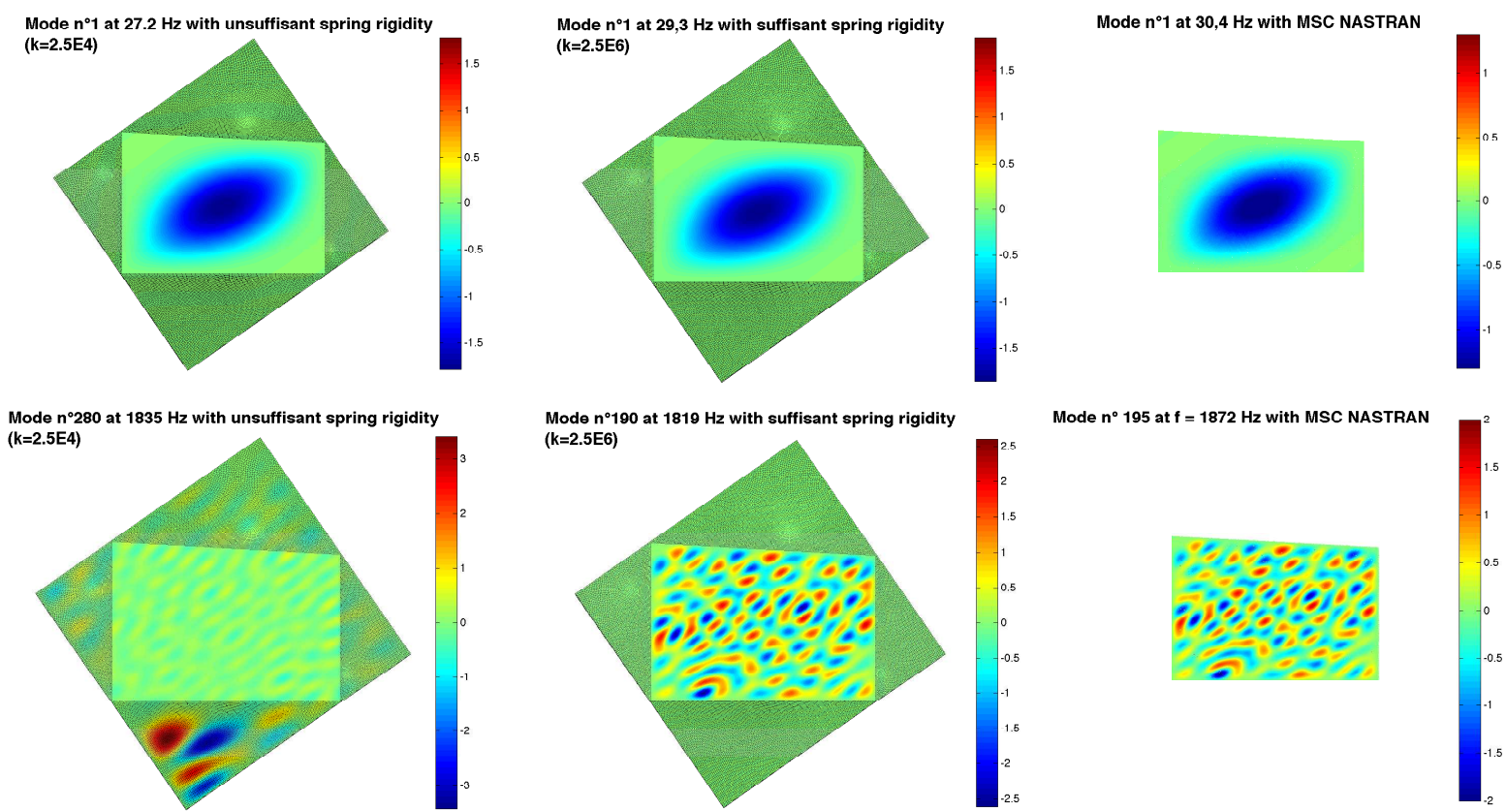

Mode $n^{\circ} 195$ at $f=1872 \mathrm{~Hz}$ with MSC NASTRAN

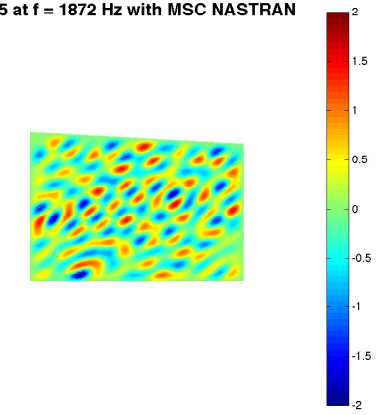

Fig. 5 - (Color online) Modal shapes of a clamped non-rectangular plate with an angle of orthotropy of $34.8^{\circ}$. Left: insufficient spring rigidity; Center: sufficient spring rigidity; Right: FEM-NASTRAN with a mesh of $5 \mathrm{~mm}$.

We also compared our modelling with a NASTRAN FEM model. This is a 2D model with a non-rectangular clamped plate meshed with orthotropic PSHELL elements.

7 The size of the elements is around $5 \mathrm{~mm}$ to ensure the convergence of the 8 calculations for the whole frequency range of interest $[0 ; 3000] \mathrm{Hz}$.

9 The comparison between our analytical model (Fig. 5, center) and numerical FEM

10 (Fig. 5, right) shows that our solution is relevant. Indeed, the clamped boundary 11 conditions, frequencies and modal shapes are well-described by our method: the

12 MAC criterion is 0.9997 and 0.948 for the modes presented in Fig. 5, respectively the 13 first modes around $30 \mathrm{~Hz}$ and another around $1.8 \mathrm{kHz}$. Concerning the frequencies 14 the difference is less than $4 \%$, which is perfectly acceptable. 


\section{III. MODELING AN UPRIGHT PIANO SOUNDBOARD}

2 In the following, we focus on the upright piano soundboard of a Pleyel P131. This

3 soundboard is made of spruce stiffened on one face by ten ribs also made of spruce

4 placed in the direction of the weak Young's modulus and on the other face by a bass

5 bridge and a main bridge (medium and high pitched notes), made of beech, placed in a

6 direction nearly parallel to the strong Young's modulus (wood fibers). There are also two

7 "cut-off" corners delimited by two beams made of beech. See Fig. 1.

\section{III.A. SUPERSTRUCTURES: RIBS AND BRIDGES}

9 The model of the superstructures is an extension of the model presented in [60].

10 Indeed, it now allows having linear superstructures of different lengths in addition to 11 different materials, widths, heights and offsets from the middle plane of the plate. Fig.

126 describes the geometry modeled. 


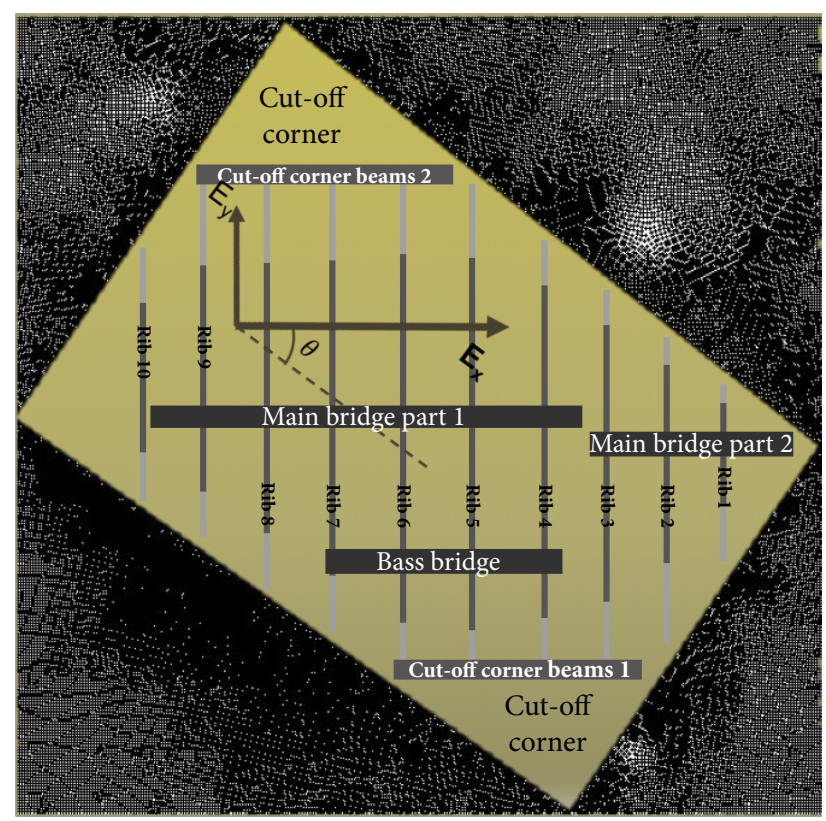

Fig. 6 - (Color online) Geometry modelled for the Pleyel P131.

3 The model developed allows having only linear superstructures parallel to the entire

4 plate edges, i.e. in the direction of the wood fibers or perpendicular to them. Taking

5 into account a curved main bridge or a stiffener that is not parallel to an extended

6 plate edge will imply expressing variable $x$ as a function of $y$ and vice-versa.

7 Therefore it will be impossible to carry out simplifications useful for maintaining an

8 analytical approach as long as possible. Thus the bridges and "cut-off" corner beams

9 become exactly parallel to the strongest Young's modulus $E_{x}$. The main bridge is cut

10 into two parts and shifted between ribs 3 and 4. Each has a constant height and

11 width. They are placed at position $y_{c}$ in the coordinates of the extended rectangular

12 plate. All the dimensions were measured on a real Pleyel P131 piano soundboard.

13 Table 2 gives the dimensions and properties of the superstructures.

14 The ribs are oriented perpendicular to the direction of the wood fibers and 15 perpendicular to the bridges to compensate for the weakness of the wood (weak 
1 Young's modulus $\left.E_{y}\right)$. Thus the ribs have a predominantly static effect. Their heights

2 are not constant along their lengths. Indeed, they are tapered at their extremities to 3 obtain flexibility near the edges of the soundboard. See Fig. 7-a.

(a)

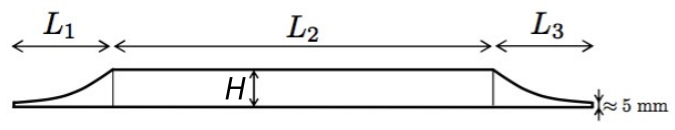

(b)

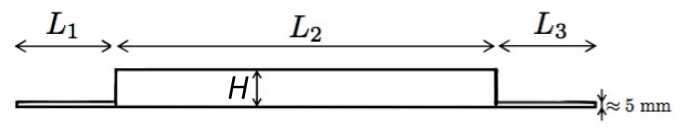

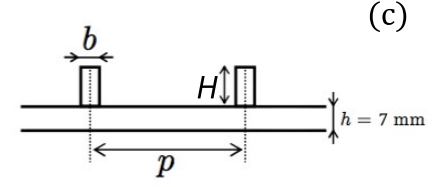

(c)

5 Fig. 7 - (Color online) Rib geometry. a) Section view of a real soundboard rib. b) Simplified rib in the 6 present model. c) Partial front view of the soundboard.

7 In our model, this progressive decrease of height is simplified. We consider a sudden 8 change of the height, as shown in Fig. 7-b. All the ribs have a height of $5 \mathrm{~mm}$ at their $9 \quad$ extremities. Table 3 gives the dimensions and properties of the ribs.

10 The boundary conditions are applied to the middle plane of the plate. The ribs and 11 bridges are bound to it through a double continuity in displacement and rotation at 12 each interface. Moreover, their heights are sufficiently low to neglect the warping 13 phenomenon. Consequently, the plate controls the global motion of the whole system 14 and its motion field can be extended to the superstructures. In addition to the 15 continuity at the interface, the motion fields in the plate / stiffener sections are 16 considered linear [60]. We also take into account bending and torsion phenomena.

17 This leads us to express the Hamiltonians of each superstructure (for more 18 information on this calculations, see Appendix A). We give the Hamiltonian for the 19 middle part of a rib (in direction $\vec{y}$ ) and of a bridge (in direction $\vec{x}$ ): 


$$
\begin{gathered}
H_{\text {rib }}=\int_{t_{0}}^{t_{1}} \frac{1}{2} \int_{x=0}^{L_{x}} \int_{y=y_{i}}^{y_{f}=y_{i}+L_{2}}\left(\left[\rho_{r}\left(I_{f} w_{, y}^{2}+b H w^{2}\right)-E_{r} I_{f} w_{, y y}^{2}\right]+\left[\rho_{r} I_{g} w_{, x}^{2}\right.\right. \\
\left.\left.\quad-G_{r} I_{g} w_{, x y}^{2}\right]\right) \delta\left(x-x_{r}\right) d x d y d t \\
H_{\text {bridge }}=\int_{t_{0}}^{t_{1}} \frac{1}{2} \int_{x=x_{i}}^{x_{f}=x_{i}+L} \int_{y=0}^{L_{y}}\left(\left[\rho_{c}\left(I_{f c} w_{, x}^{2}+b_{c} H_{c} w^{2}\right) \cdot-E_{c} I_{f c} w_{, x x}^{2}\right]+\left[\rho_{c} I_{g c} w_{, y}^{2}\right.\right. \\
\left.\left.-G_{c} I_{g c} w_{, x y}^{2}\right]\right) \delta\left(y-y_{c}\right) d x d y d t
\end{gathered}
$$

1 Table 4 summarizes all the constants used in the previous equations. As in Eq. (1),

2 the entire Hamiltonian for the whole system (with $N_{r}$ being the number of ribs and

3 extremities and $N_{b}$ the number of bridges, for which all the constants and positions

4 are different) becomes:

$$
H_{\text {soundboard }}=H_{o p}+\sum_{r=1}^{N_{r}} H_{r i b}^{(r)}+\sum_{b=1}^{N_{b}} H_{\text {bridge }}^{(b)}
$$

5 and the eigenvalue matrix problem as described in section II.A.2:

$\left\{\overline{\bar{M}}^{\text {plate }}+\overline{\bar{M}}^{\text {springs }}+\overline{\bar{M}}^{\text {ribs }}+\overline{\bar{M}}^{\text {bridges }}\right\} \ddot{\vec{a}}+\left\{\overline{\bar{K}}^{\text {plate }}+\overline{\bar{K}}^{\text {springs }}+\overline{\bar{K}}^{\text {ribs }}+\overline{\bar{K}}^{\text {bridges }}\right\} \vec{a}=\overrightarrow{0}$

6 Therefore we determine the new transfer matrix $\overline{\bar{T}}$ of the Pleyel P131 and the 7 generalized matrix formulation with a second member on the basis of the piano 8 modes:

$$
-\omega^{2} \overline{\bar{M}}_{\text {soundboard }} \vec{b}(\omega)+j \omega \overline{\bar{C}} \vec{b}(\omega)+\overline{\bar{K}}_{\text {soundboard }} \vec{b}(\omega)=\overline{\bar{T}}^{t} \vec{F}_{\text {gen }}
$$


$1 \quad$ With $\quad \overline{\bar{M}}_{\text {soundboard }}=\overline{\bar{T}}^{t}\left\{\overline{\bar{M}}^{\text {plate }}+\overline{\bar{M}}^{\text {springs }}+\overline{\bar{M}}^{\text {ribs }}+\overline{\bar{M}}^{\text {bridges }}\right\} \overline{\bar{T}} \quad$ and $\quad \overline{\bar{K}}_{\text {soundboard }}=$ $2 \quad \overline{\bar{T}}^{t}\left\{\overline{\bar{K}}^{\text {plate }}+\overline{\bar{K}}^{\text {springs }}+\overline{\bar{K}}^{\text {ribs }}+\overline{\bar{K}}^{\text {bridges }}\right\} \overline{\bar{T}}$. Damping matrix $\overline{\bar{C}}$ is the same as that 3 presented in Sec. II.A.3.

\section{III.B. ACOUSTIC RADIATION OF A NON-RECTANGULAR RIBBED STRUCTURE}

5 The method we propose for acoustic radiation is an alternative to a purely numerical

6 method such as the finite element boundary method, Rayleigh integral or Perfectly

7 Matched Layers. Thus we calculate the acoustic radiation of the structure using the

8 radiation impedances of the extended rectangular un-ribbed plate, which has been

9 studied several times by Wallace, Maidanik and Stepanishen [34], [31], [30], for

10 example. This approach is currently limited to a rectangular ribbed plate [63] but we

11 propose to extend it to the case of a non-rectangular structure. In addition, this

12 approach allows comparing the radiation modal impedances of a non-rectangular

13 ribbed structure to those of an equivalent simple plate, as was done in our previous

14 article [60]. This could be particularly interesting in the frequency range where the

15 modes are similar to un-ribbed plate modes (i.e. at low frequencies).

16 We can consider that the piano case is an obstacle to acoustic short circuiting past

17 the first modes. At low frequency, this results in a decrease of radiation [26], [32]. On

18 the contrary, when focusing on frequencies higher than the first octave, as will be the

19 case in the following, the wavelengths quickly become small enough to consider a

20 baffled hypothesis. Note that this hypothesis does not change the tendencies of the

21 results and has no repercussion on the parametric studies. 
1 The light fluid assumption is also made as it implies omitting inter-modal coupling

2 brought by the action of a fluid on the structure. Several authors have shown that

3 these couplings are negligible at the first order [31], [68], [29] or do not change the

4 general tendencies [69], [41], [54]. However, other authors have shown that some

5 changes can occur in the case of heterogeneous boundary conditions, high

6 superstructures or at resonances [70], [71]. We assume that they are neglected and

7 thus assign the acoustic radiated power $W(\omega)$ of the piano soundboard in the

8 frequency domain as [60], [29]:

$$
W(\omega)=\frac{\omega^{2}}{2} \vec{b}^{t} \overline{\bar{T}}^{t} \overline{\bar{R}}(\omega) \overline{\bar{T}} \vec{b}
$$

9 with $\overline{\bar{R}}(\omega)$ being the real part of the inter-modal acoustical impedance matrix.

10 Due to the light fluid assumption this matrix is diagonal, i.e. inter-modal couplings are 11 neglected. Thus the diagonal terms are calculated numerically and defined as [26], 12 [29]:

$$
R_{m n m n}(\omega)=\frac{\rho_{0} \omega}{\pi^{2}} \iint_{0}^{k} \frac{\left|\tilde{\phi}_{m n}\left(k_{x}, k_{y}\right)\right|^{2}}{\sqrt{k^{2}-k_{x}^{2}-k_{y}^{2}}} d k_{x} d k_{y}
$$

13 where $\tilde{\phi}_{m n}\left(k_{x}, k_{y}\right)$ refers to the bi-dimensional Fourier transform of a simply 14 supported rectangular baffled plate mode.

16 The vibro-acoustical behavior of the piano soundboard can be split into different 17 frequency ranges in which the vibration and radiation are different, due to the design 
1 of the instrument. In the following, we will first focus on the modal aspects of the

2 instrument and then on the vibro-acoustical response of the soundboard.

3 To ensure the convergence of the calculations, the truncation order on the modal

4 basis is overestimated and, as with the unribbed plate, fixed at $(M, N)=(82,63)$.

5 Moreover in order to validate the blocking condition along the non-rectangular

6 contours, the same studies as shown in Figs 4 and 5 were performed (spring rigidity 7 and number).

\section{III.C.1. SAMPLES OF UPRIGHT PIANO SOUNDBOARD MODES}

In terms of modal shapes, many phenomena appear as a function of the frequency range considered. Indeed, the more the frequency increases, the higher the rigidity of the superstructures is. Therefore the superstructures will strongly limit the transverse displacement of the soundboard when the frequency increases. This begins with the strongest of superstructures, i.e. the bridges, and then the ribs.

14 Some studies have also shown that the vibration is localized in certain frequency

15 ranges. Waveguide effects occur when the wavelengths reach the same order of magnitude as the inter-rib spaces [17], [18]. Fig. 8 shows a classification of modal shapes for the 200 first modes (below $3 \mathrm{kHz}$ ). We define four families of modes:

- "Similar" to plate modes, i.e. unribbed modes that can be named with the number of half wavelengths in the different directions (mode $(3,1)$ for example);

- Blocking bridges only, when the two bridges strongly minimize the plate transverse displacement; 
- Blocking bridges and ribs, when all the superstructures strongly minimize the plate transverse displacement;

- Localized vibrations, when the vibration is localized in a small area delimited by the superstructures.

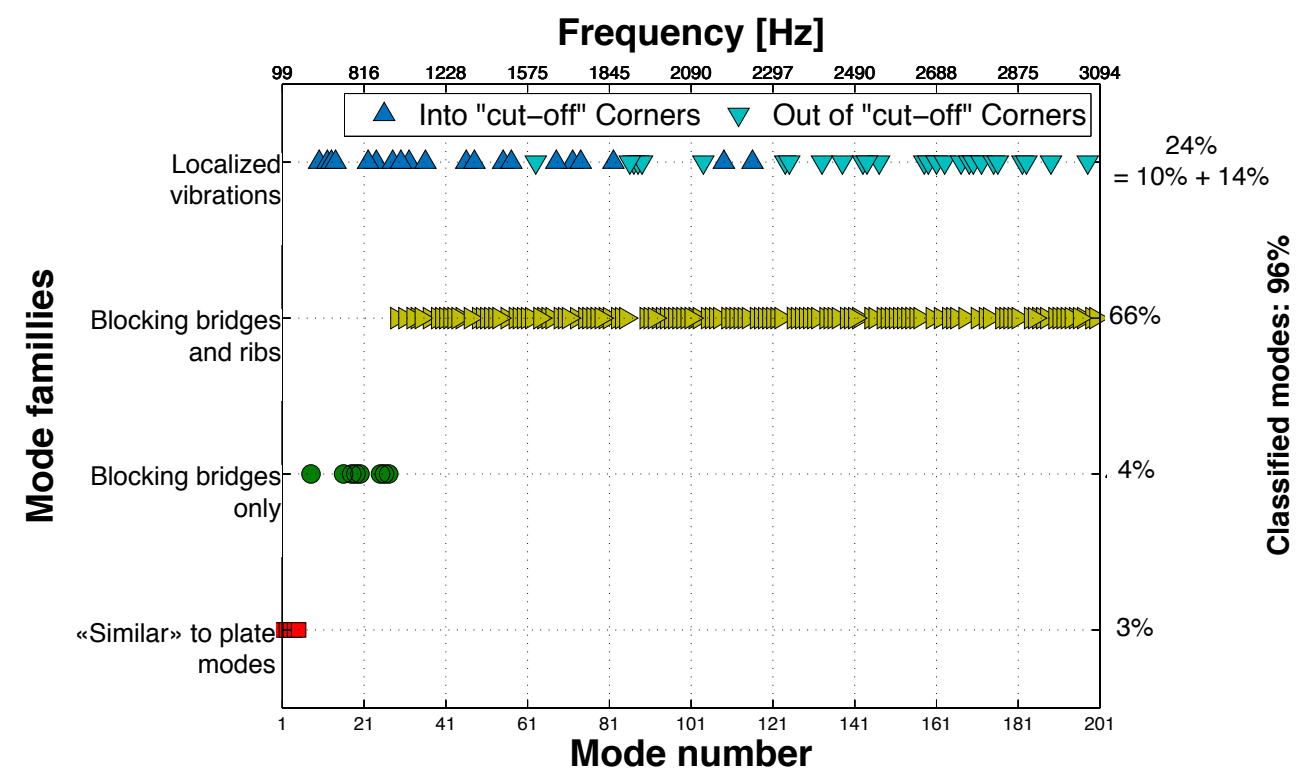

Fig. 8 - (Color online) Classification of the first 200 modes of a Pleyel P131 soundboard.

To classify the modal shapes into these different families, we use an average quadratic linear velocity to evaluate numerically when the vibration is reduced at the stiffener locations. This allows determining the points at which the transverse displacement is strongly limited. To do this, we define a velocity threshold that allows separating a sample of modes classified visually previously (around 20 modes). Regarding the "localized vibrations" family, which is a sub-family of "blocking bridges and ribs", a criterion of spatial distribution is added. We first detect the maximum velocity magnitude of the structure and then compare the entire space average quadratic velocity with the space average quadratic velocity 
on a smaller surface around the maximum magnitude determined previously. We gradually increase the surface so that the local average velocity becomes higher than a certain percentage of the entire space average velocity. Similarly, this percentage is determined with a sample of modal shapes classified visually. In this way, we can classify around $73 \%$ of the modal shapes that are checked visually as a final control. The unclassified modes are determined visually and we finally classify $96 \%$ of the modes into the four families.

We can see that the ratios between them are very different. Indeed, for a large majority of modes all the superstructures strongly minimize the transverse displacement (sum of triangle markers around $90 \%$ ), whereas the modes "blind" to the superstructures amount to only around 3\% (see the square markers on Fig. 8). $(3,1)$. This phenomenon is limited to the five first modes of the soundboard.
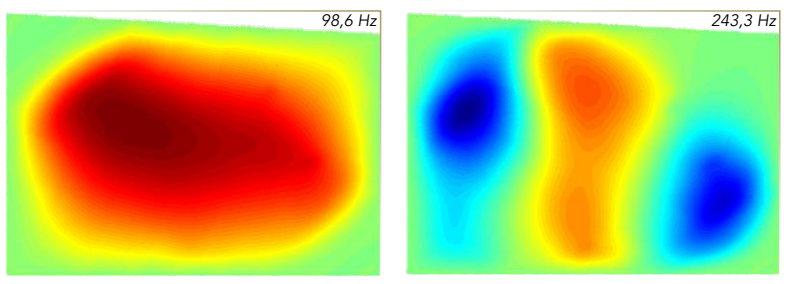

Fig. 9 - (Color online) Low frequency modes similar to unribbed plate modes.

A second family can be associated with the frequency range $[300 ; 900] \mathrm{Hz}$ when the bridges (the strongest superstructures) strongly minimize the transverse 
displacement of the plate. This family is illustrated in Fig. 10 and matches with the circle markers in Fig. 8.
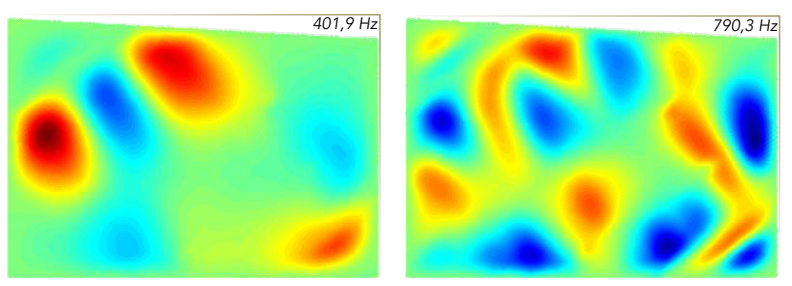

Fig. 10 - (Color online) Modes where the transverse displacement is strongly minimized by the bridges.

For the majority of modes (higher than $1 \mathrm{kHz}$ ), all the superstructures strongly minimize the transverse displacement. For around $66 \%$ of them, the vibrations are spread over the whole plate or at least over a large area (see the triangle markers pointing to the right in Fig. 8). Fig. 11 shows two of these modes, the first around 1 $\mathrm{kHz}$ and the second around $3 \mathrm{kHz}$ : the location of the superstructures can be easily guessed.
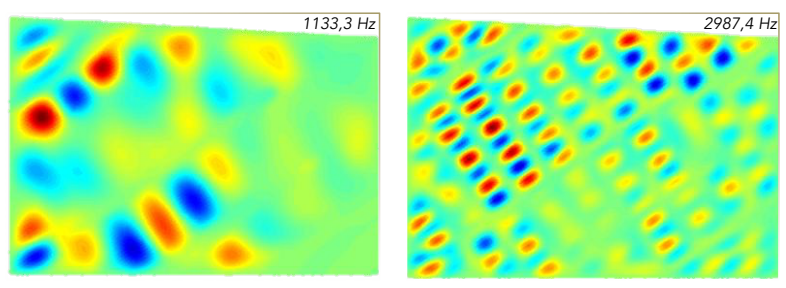

Fig. 11 - (Color online) Modes where the transverse displacement is strongly minimized by all the superstructures.

In addition, the vibrations are also localized in areas delimited by superstructures for the last $24 \%$. It is important to split these $24 \%$ into two sub-parts: $10 \%$ of "cutoff" corner modes (upward triangle markers in Fig. 8) and 14\% of vibrations 
localized between the ribs (downwards triangle markers in Fig. 8). Fig. 12 shows two examples of this $14 \%$ of modes.
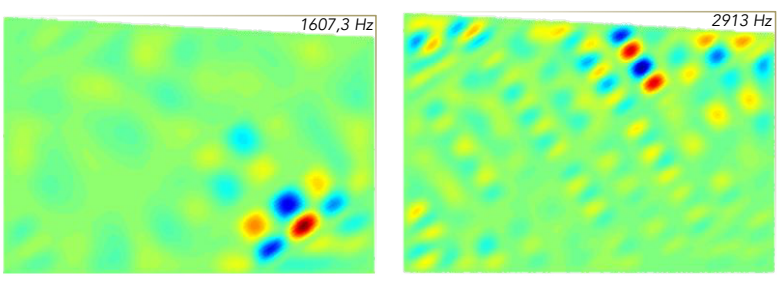

Fig. 12 - (Color online) Modes where the vibrations are localized into areas delimited by superstructures.

This phenomenon of localization between the ribs seems to begin at around 1600 $\mathrm{Hz}$, thus when the wavelengths are around the same order of magnitude as the inter-rib spaces [17], [18]. But as shown in Fig. 12, the percentage of this family is quiet low compared to the "blocked modes" (only $14 \%$ vs. $67 \%$ ): the wavelength condition seems to be a necessary but not sufficient to localize the vibrations in a reduced area of the soundboard.

Finally, the last $4 \%$ are unclassified modes: the bridges begin to minimize the transverse displacement but not completely.

\section{III.C.2. MobILITY, SPACE AVERAGE QUADRATIC VELOCITY AND}

\section{ACOUSTIC RADIATED POWER ALONG THE BRIDGE}

The mechanical mobility at the bridge is an essential quantity in musical acoustics because it characterizes the transfer between the source and the radiator. Several piano soundboard studies exist on the subject [11], [18]. In the case of a harp soundboard, maps of mobility along the soundboard (where the points chosen for 
the excitation correspond to the coupling points between the soundboard and the strings) as function of the frequency have been performed by [61], [62]. However, the utility of mobility is limited to the local vibration at the excitation point. In addition, we think it is worthwhile completing the analysis with other indicators, such as space average quadratic velocity and acoustic radiated power, which take into account the entire vibration of the soundboard. In this way, we consider an excitation located on different points of the main bridge, corresponding to the string coupling points ranging from note 32 ( $E 3$ with a fundamental frequencyof $f_{0}=164.8$ $\mathrm{Hz})$ to $76\left(\mathrm{C} 7\right.$ with $\left.\mathrm{f}_{0}=2093 \mathrm{~Hz}\right)$, so from the left to the right of the entire main bridge (parts 1 and 2) in Fig. 6.

Fig. 13 shows, from top to bottom: the mobility, the space average quadratic 13 plotted with the same dynamic of $60 \mathrm{~dB}$.

14 Note that in the case of a harmonic problem when considering the modal 15 decomposition Eq. (2) and the relation between the initial and piano basis Eq. (5), 16 the space average quadratic velocity in the soundboard area is given by the 17 following expression [29]:

$$
\left\langle v^{2}(\omega)\right\rangle=\frac{L_{x} L_{y}}{S_{\text {int }}} \frac{\omega^{2}}{8} \sum_{m=1}^{M} \sum_{n=1}^{N}\left|a_{m n}(\omega)\right|^{2}=\frac{L_{x} L_{y}}{S_{\text {int }}} \frac{\omega^{2}}{8} \vec{b}^{t}(\omega) \overline{\bar{T}} t \overline{\bar{T}} \vec{b}(\omega)
$$
where ${ }^{t}$ refers to the transpose matrix or vector and ${ }^{*}$ to the complex conjugate. $S_{\text {int }}$ 19 is the surface of the soundboard, i.e. the unblocked surface. Regarding the 20 radiated acoustic power, its expression was given in Eq. (17). 

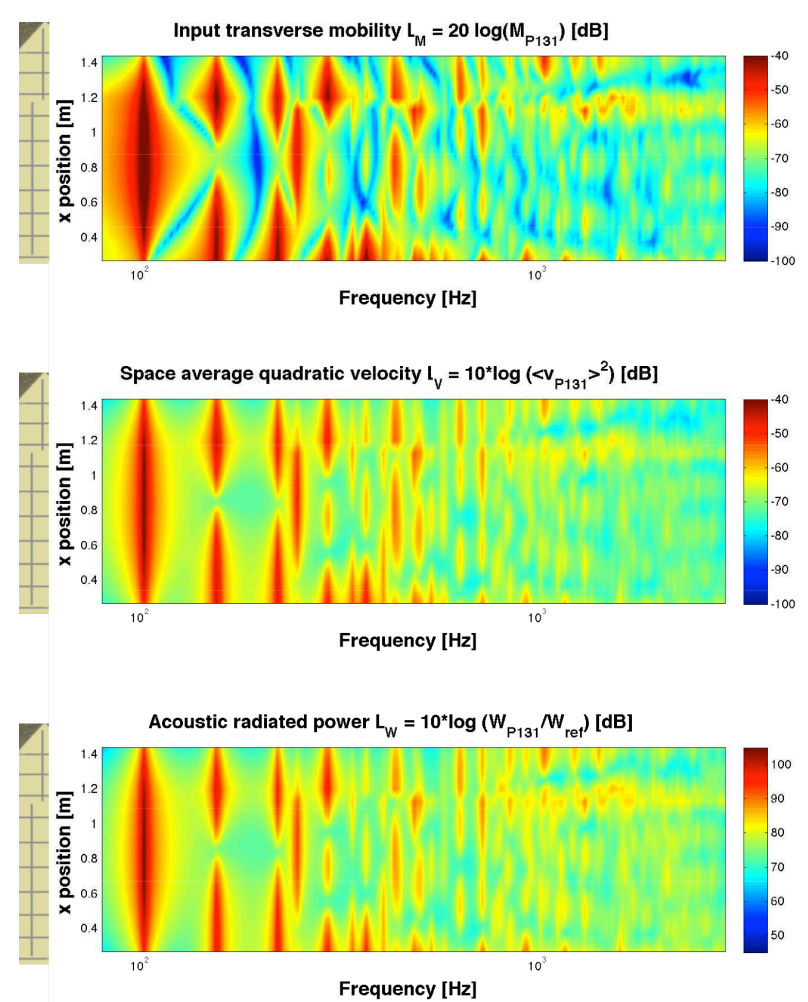

2 Fig. 13 - (Color online) Evolution of local and general indicators at string/main bridge coupling points: Top: mobility; Center: space average quadratic velocity; Bottom: Acoustic radiated power. Globally, the three indicators follow the same trends. Firstly, dB levels tend to decrease when the frequency increases. Moreover, the strings coupled near the extremity and near the cut-off of the main bridge are sensitive to the local and sudden change of the bridge height. Thus, there is an increase of mobility, space average quadratic velocity and acoustic radiated power around $0.3 \mathrm{~m}$ and $1.2 \mathrm{~m}$ in Fig. 13, i.e. near the beginning of the bridge and near its break. Secondly, in the frequency range $[400 ; 3000] \mathrm{Hz}$ the dynamic level is around $1 / 3$ lower than $400 \mathrm{~Hz}$. This becomes more apparent at high frequency when the modal overlap masks the mode resonances. 
However, the dynamic level of the mobility is higher than the others, regardless of the frequency range (see Fig. 13). This is consistent because mobility is a local indicator whereas the others take into account the entire behavior of the soundboard. Below $400 \mathrm{~Hz}$, the mobility fluctuates around $65 \mathrm{~dB}$ whereas the space average quadratic velocity and the radiated power only around $40 \mathrm{~dB}$, so around $1 / 3$ lower. Above $400 \mathrm{~Hz}$, the ratio is around the same order of magnitude.

\section{III.C.3. MOBILITY AND ACOUSTIC RADIATED POWER FROM THE MUSICAL}

\section{VIEWPOINT}

From the musical viewpoint, the large quantity of information in this kind of presentation makes it difficult to interpret the results. Indeed, the response of an instrument is not continuous in frequency but discrete, in addition to the discrete variation of the excitation position. In the case of a harp soundboard, [61], [62] focused on admittance at discrete frequencies corresponding to string fundamentals. These authors also did the same for the first and the second partials, neglecting the inharmonicity brought about by mobility, i.e. by simply multiplying the fundamental frequencies. In the following, we extend this idea to

17 the acoustic radiated power. This study is limited to partials due to string 18 transverse waves below $3 \mathrm{kHz}$. We present the average values of each indicator and the gradients between successive notes (in absolute values) from the $32^{\text {th }}$ to $76^{\text {th }}$. All the partials have the same magnitudes. We give the following expressions for the average values of mobility and acoustic radiated power: 


$$
\begin{aligned}
& L_{M}(\text { key })=20 \log \left(\frac{1}{N_{\text {partials }}} \sum_{n=1}^{N_{\text {partials }}} \frac{\left|\dot{w}\left(x_{\text {key }}, y_{\text {key }}, n f_{\text {key }}\right)\right|}{F}\right) \\
& L_{w}(\text { key })=10 \log \left(\frac{1}{N_{\text {partials }}} \sum_{n=1}^{N_{\text {partials }}} \frac{W\left(x_{\text {key }}, y_{\text {key }}, n f_{\text {key }}\right)}{W_{\text {ref }}}\right)
\end{aligned}
$$

1 Obviously, we are still far from a realistic force because the different partials do not

2 have the same amplitudes in reality. However, the advantage of these indicators is 3 that they present "pictures" of pianos, making it possible to compare different $4 \quad$ instrument designs.

5 In all the following figures, the X-axis limits are the same but the labels are 6 different. That of the radiated power corresponds to the note numbers with the 7 related note names whereas that of the mobility corresponds to the fundamental 8 frequencies of each note. Moreover, all these figures are plotted with the same 9 dynamic in order to compare the variation of each indicator. 

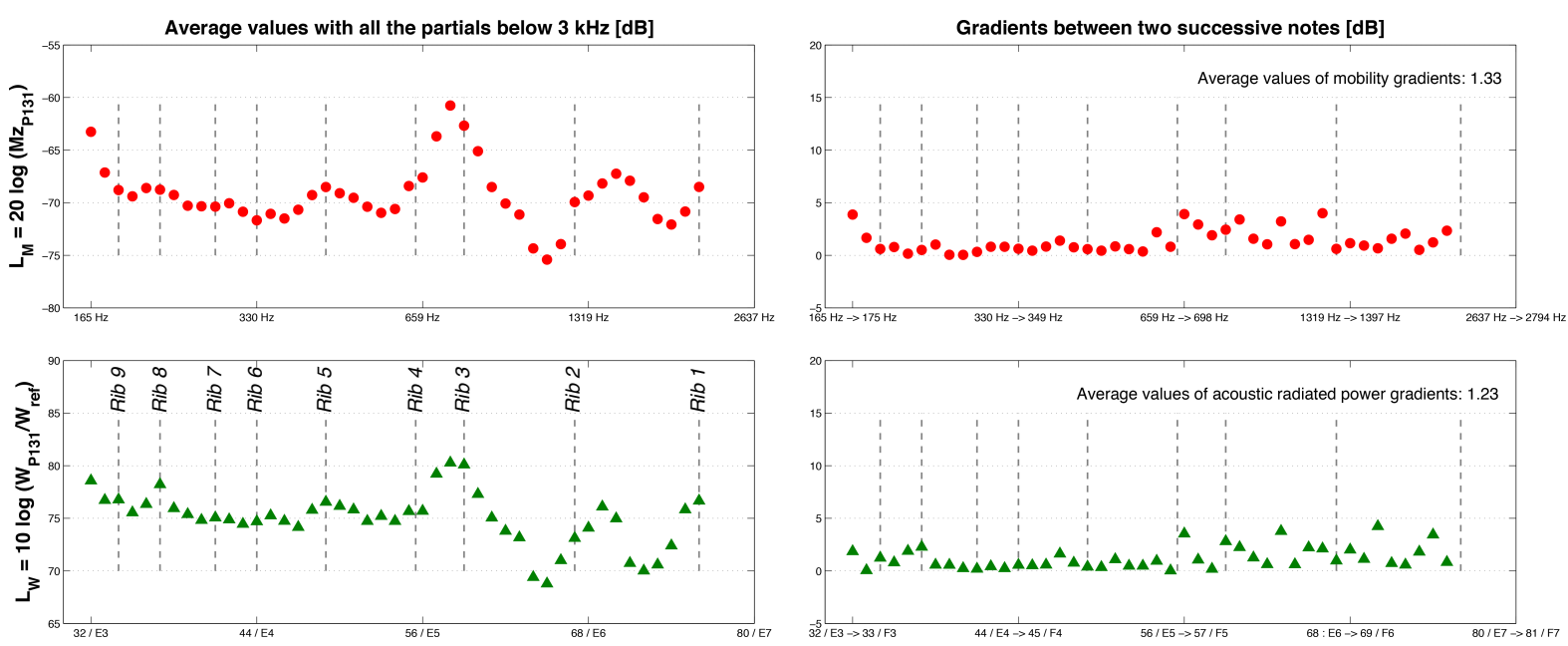

Fig. 14 - (Color online) Evolution of mobility (top) and acoustic radiated power (bottom) of a P131 soundboard. Left: dB values of indicators; Right: gradients (absolute values) between two successive notes in $\mathrm{dB}$.

Up to now, we have only focused on the results relating to the P131 soundboard (Fig. 14). A major tendency is the inhomogeneity of mobility and power levels along the bridge (left Fig. 14), particularly at the bridge break between notes 58 and 59, where both increase considerably. The same applies for the notes near the beginning of the main bridge (near notes 32 ) where $\mathrm{dB}$ levels have the same The inhomogeneity of the response along the bridge is not limited to its beginning and break. Regardless of the indicators, focusing on discrete frequencies also shows that the higher variations appear after the bridge break, which is not

14 obvious on the mobility map view in Fig. 13. Above note 59, the wavelengths become small and the waves no longer pass through the bridge break, contrary to waves with lower frequencies. Therefore the second part of the main bridge 
(almost half the size of the first part of the main bridge, see Fig. 6) cannot distribute the vibration over the whole soundboard.

Moreover, the latter notes suffer from poor harmonic richness that maximizes the variations. Indeed, in the case of the first notes, the large number of harmonics will average the soundboard response, partly masking this phenomenon. Thus our model shows that the higher the order of notes is, the greater the variability may be.

This raises the question of the "killer-octave" which is, according to piano makers, around the penultimate octave (C6 to $\mathrm{C} 7$ ). Fandrich [72] describes it as a region in the fifth to sixth octave (C5 to $\mathrm{C6}$ ) of the keyboard. In both cases, it is characterized by low sustain and is present in most pianos regardless of construction method or soundboard design. Our results show two areas that could correspond to this phenomenon: the first with a large increase of the mobility and

14 the acoustic radiated power; the second their decrease.

15 Focusing only on the average values would lead to an incomplete study. Indeed, in 16 a playing situation, a major variation between two consecutive notes implies 17 considerable perceptive inhomogeneity for the pianist and the listener. With this in 18 mind, the gradients between successive notes provide much interesting 19 information (right, Fig. 14). They show considerable variability between two 20 successive notes and thus heterogeneous playing: the average mobility and 21 acoustic radiated power gradients are around 1.33 and $1.23 \mathrm{~dB}$, respectively. 
The soundboard response can also be split into two ranges of notes. For the third and fourth octaves, the note-to-note gradients are low and the responses are globally homogeneous, which is also the case for both the mobility and the acoustic radiated power. Above the beginning of the fifth octave at around $659 \mathrm{~Hz}$, the gradients increase, with higher values of around $4 \mathrm{~dB}$. Thus, for high-pitched notes, it is common to have a perceived acoustic power division or multiplication by 2.5 compared to the previous notes. The same effect occurs near the beginning of the bridge but to a lesser extent. Note that a significant mobility gradient does not systematically mean the same for the acoustic radiated power and vice-versa. Moreover, we do not find a regular relationship between a decrease of the indicators and the location of the ribs (represented by dashed lines in Fig. 14): sometimes a coupling close to a rib tends to decrease the three indicators and sometimes it tends to increase them. To review, the behavior can be split into two main ranges: after the bridge break, where the note-to-note gradients are higher and where there are major global variations of mobility and acoustic radiated power; before the bridge break where global variations of mobility and radiated power also exist but are lower. Highpitched notes (and the first notes) seem to be particularly difficult to control by piano makers, contrary to medium notes.

Applying a force at the bridge tends to distribute the force over the entire plate and thus homogenize the piano soundboard response. In the case of discontinuities, this distribution effect is broken if the discontinuity is significant enough compared 
to the wavelength of the wave propagated: at low frequencies, the wavelengths are long and the discontinuity has no impact on the propagation; at high frequencies, the wavelengths are short and a discontinuity will be an obstacle to the propagation of waves.

Finally, as with all string instruments, piano strings have multiple modal frequencies called partials or harmonics. For $32^{\text {th }}$ note, there are 18 frequencies below $3 \mathrm{kHz}$, whereas there is only one for the last note studied, number 76 . Thus a discontinuity could have a higher influence on high-pitched notes and on the high order harmonics of the first notes. Consequently, it seems that if a bridge break is necessary in the design of the instrument it could be placed at low frequency to minimize its effect. On the contrary, the removal of any break at the bridge would homogenize the response of the instrument.

\section{III.D. EXPERIMENTAL AND NUMERICAL COMPARISONS}

To confirm the hypothesis made in our model, some comparisons were made with the Nastran FEM method and experimental results. The measurements were performed in the framework of an internship Master degree [73]. We present several results in this paper to validate the hypothesis made in our model. The Pleyel P131 soundboard used was separated from the case and clamped to a solid wall made of concrete to recreate baffled conditions. The structure was excited at the bridge in the treble zone with a white noise and the vibration measured on the rib side with a POLYTEC PSV-400 vibrometer laser with a mesh of 4600 points. Despite the geometrical simplifications in our model, the experimental results showed good 
agreement between the modal shapes and those of our model, as shown by the

2 images in the top row in Fig. 15, for low frequency modes. In addition, the lower

3 images in Fig. 15 give examples of high order modes around $2 \mathrm{kHz}$, where the $4 \quad$ superstructures strongly minimize the vibrations.

Analytical modelling
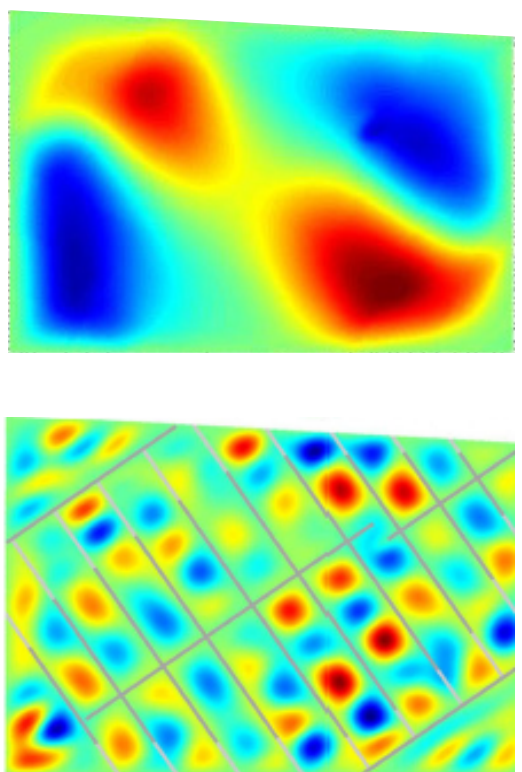

Experimental results
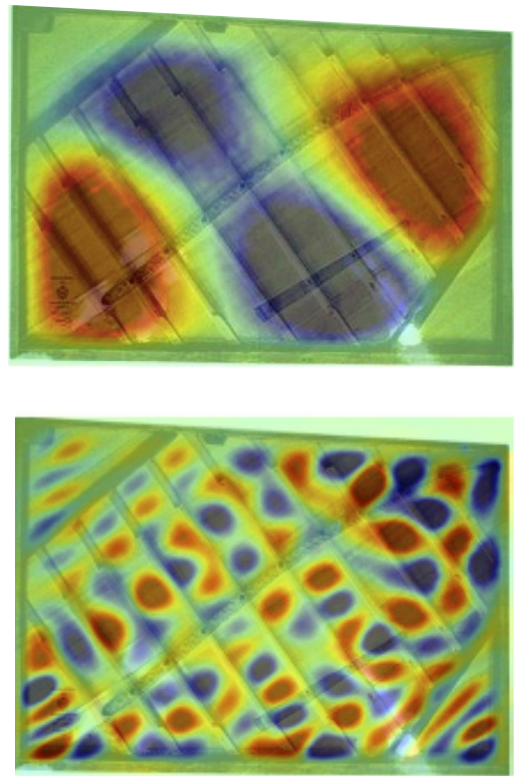

Fig. 15 - (Color online) Comparison of Pleyel P131 modal shapes between our analytical modelling and experimental results.

8 However the first eigen-frequencies were higher in our model: the experimental

$9 \quad$ eigen-frequency was around $66 \mathrm{~Hz}$ whereas that of the analytical model was around

$10100 \mathrm{~Hz}$. Therefore the acoustic radiation was overestimated around the first modes

11 without, however, changing the general trends. These differences highlight that the

12 structure in our model was too rigid. This was not due to geometrical simplifications

13 (superstructures parallel to plate edges, rib heights, etc.) since the spatial aspects

14 (modal shapes) were well-described and compared with the measurements. 
1 However, membrane effects may have had an impact on the eigen-frequencies of the

2 first modes and quickly disappeared. To determine whether this was the case, we

3 compared the experimental and our own results with a Nastran FEM model with the

4 same geometrical simplifications. In order to confirm the geometrical simplifications,

5 they were also simulated in the FEM model. The plate was meshed with PSHELL

6 elements and the superstructures with PBEAM elements deported on each face. The

7 FEM model is shown in Fig. 16 in which we consider two cases: the first with a

8 blocked membrane imposing no displacements of the plate elements; the second $9 \quad$ with a membrane effect.

a)

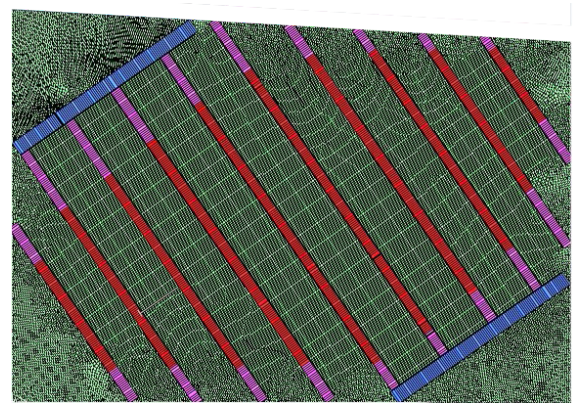

c)

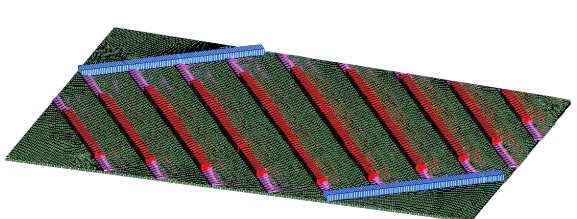

b)

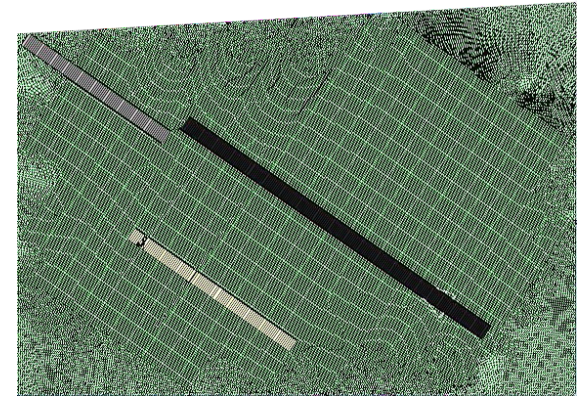

d)

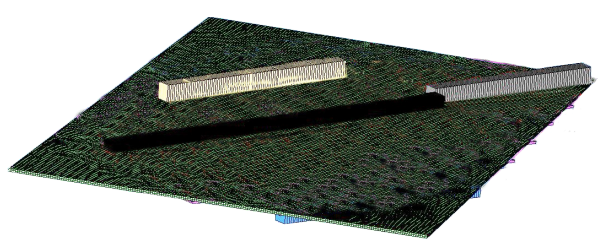

11 Fig. 16 - (Color online) Nastran FEM model of Pleyel P131 soundboard with the same geometrical

12 simplifications as in our model. a) and c) view of ribs view with cut-off corner beams; b) and d) bridge view.

14 To ensure the convergence of the calculations, the size of the finite elements must be

15 at least $1 \mathrm{~mm}$ due to the addition of stiffeners (see Fig. 17). On the contrary, the same 16 figure shows that it is not useful to increase the order of truncation in our model even 
1 if it slightly increases the precision of our calculations. This result also shows that

2 both models converge to the same solution via two different paths: our model

3 converged from the top (increasing the order of truncation provides flexibility)

4 whereas the FEM model converged from the bottom.

5

6

7

14 frequencies.

15 These comparisons show that the geometrical simplifications were relevant.

16 Membrane effects have an influence on the first eigen-frequencies and thus imply an

17 overestimation of the acoustic radiation at low frequencies. However, it is important to

18 keep in mind that the aim of this model is to perform parametrical studies. Therefore, 19 even if the first frequencies were higher than those of the real soundboard, the 

made with our model.
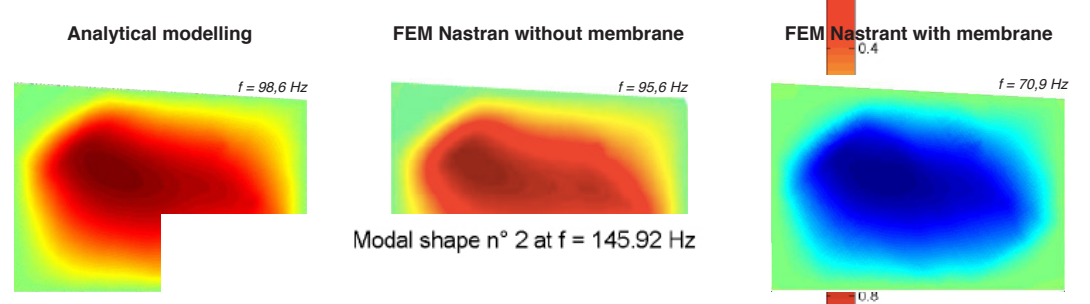

Experimental results
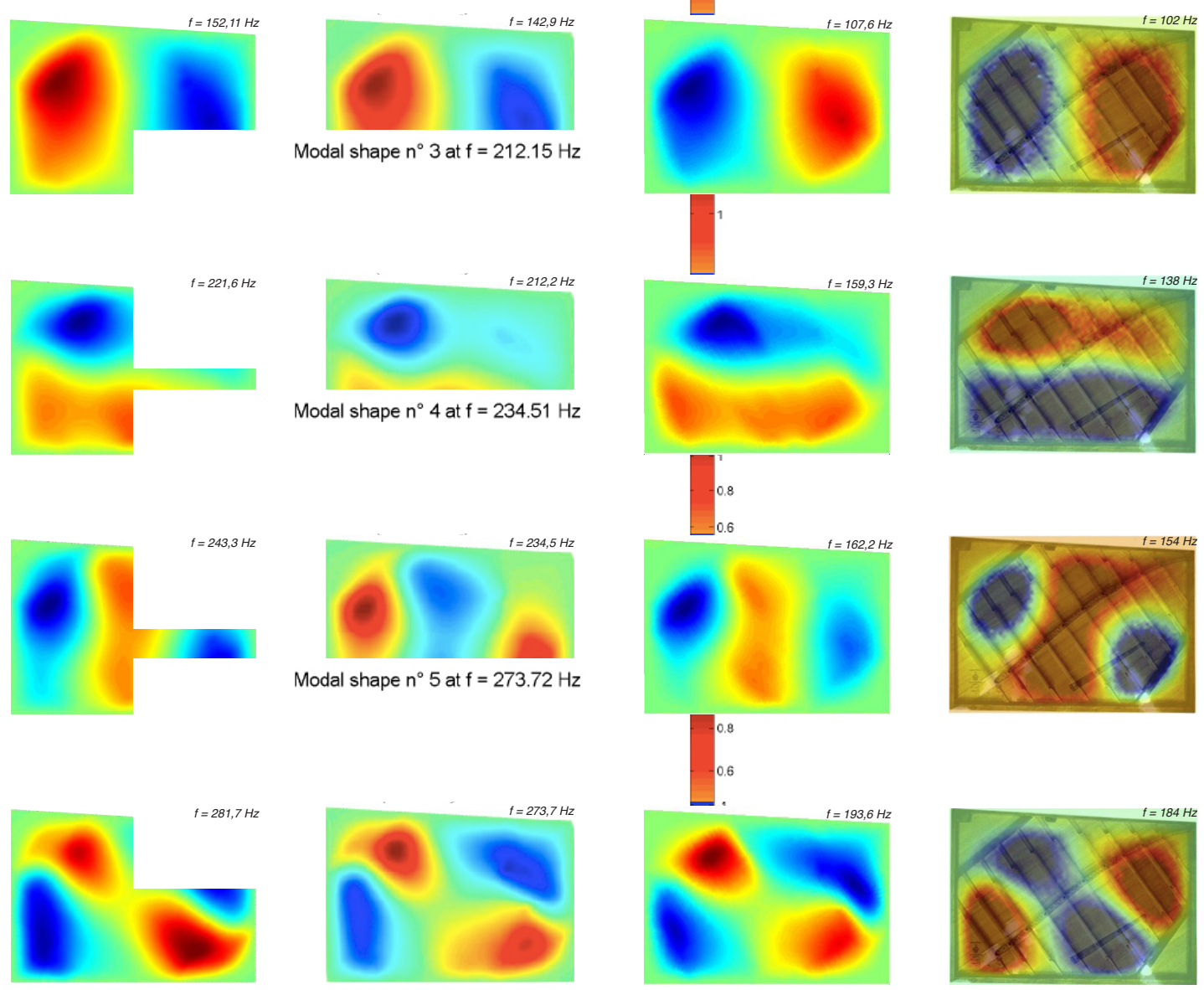

4 Fig. 18 - (Color online) Comparison of numerical and experimental modal shapes using four methods.

5 From left to right: analytical modelling, Nastran FEM without membrane, Nastran FEM with membrane and experimental results.

7 Obviously, these comparisons assume a flat soundboard and not one in a playing

8 situation. In practice the soundboard is manufactured with an initial crown in order to 

ensure the contact between the strings and the bridges. Applying and tuning the strings have two consequences: the initial crown lowers and compression stresses

3 are applied to the plate edges. This phenomenon is known as "downbearing" and

4 implies an increase of the first eigen-frequencies, as was shown in [74] and [67]

5 where we can find crossed comparisons of [4], [8], [74]-[77].

6 However, simple reasoning leads to conclusions to the contrary. Indeed, lowering the 7 crown implies decreasing the eigen-frequencies [78]. The same effect occurs when 8 compression stresses are applied to the plate [79], [64]-[66]. In both cases, it quickly

9 becomes negligible when the frequency increases. To explain this, it seems that the 10 initial crown produced by manufacturing implies a non-linear evolution of the first 11 eigen-frequencies of the soundboard. Thus the latter can increase or decrease

12 depending on the initial crown: the consequences depend on the soundboard [80].

13 Nevertheless, neglecting downbearing effects does hinder parametrical studies in the

14 same as membrane effects: the frequency shift will be around the same order of 15 magnitude in all the following studies.

\section{IV. Sensitivity to StRUctural ChanGeS}

17 From subsection III.C.3, we know that structural heterogeneities imply variations of the

18 piano soundboard response, probably leading to a discontinuous timbre or perceived

19 sound, which is a difficulty expressed by piano makers. Although the components of the 20 force injected at the coupling point with the bridge do not have the same magnitudes,

21 we think that ensuring a linear or progressive evolution of each indicator presented in 22 III.C.3 will be interesting from the musical viewpoint. At least, their variations and 
1 especially the gradients should be greatly reduced in order to obtain more

2 homogeneous playing.

3 Therefore we wanted to see how the vibro-acoustic behavior is modified through

4 structural changes. Hence we now consider two additional cases illustrated in Fig. 19:

5 Pleyel P131 soundboard with a continuous main bridge; P131 soundboard with ribs 1

6 and 2 removed. These cases are compared to the reference case presented previously

7 in III.C.3. In order to simplify the reading, solid lines are used instead of markers

8 although we continue to focus on a spectrum of discrete frequencies.

14 Obviously, a continuous main bridge implies eliminating the defect between note 15 numbers 58 and 59 because there is no longer any abrupt and local change of 16 stiffness (Fig. 20, left). Moreover, the waves are better spread all over the

17 soundboard because small wavelengths are no longer blocked by the bridge break.

Fig. 19 - (Color online) Different sets of upright piano soundboards based on the Pleyel P131 configuration. a) Reference case: Pleyel P131; b) Continuous main bridge; c) Removal of ribs for highpitched notes.

\section{IV.A. Continuous main BRIDGE}
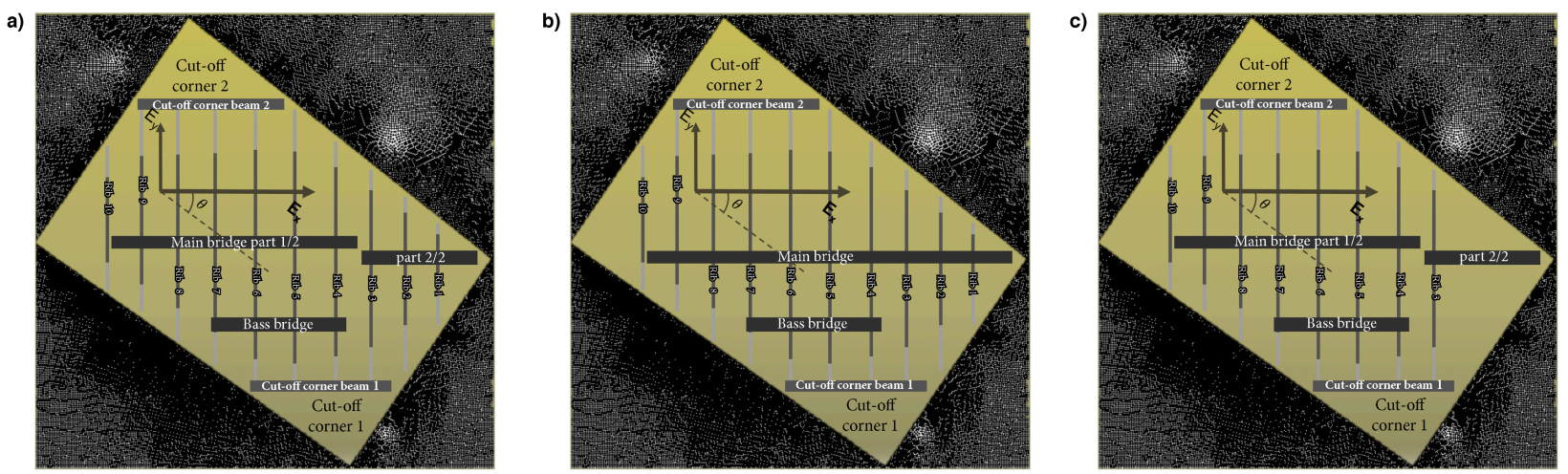
1 Thus the global variations above note 56 are now lower than in the reference case 2 (left Fig. 14), regardless of the indicators.
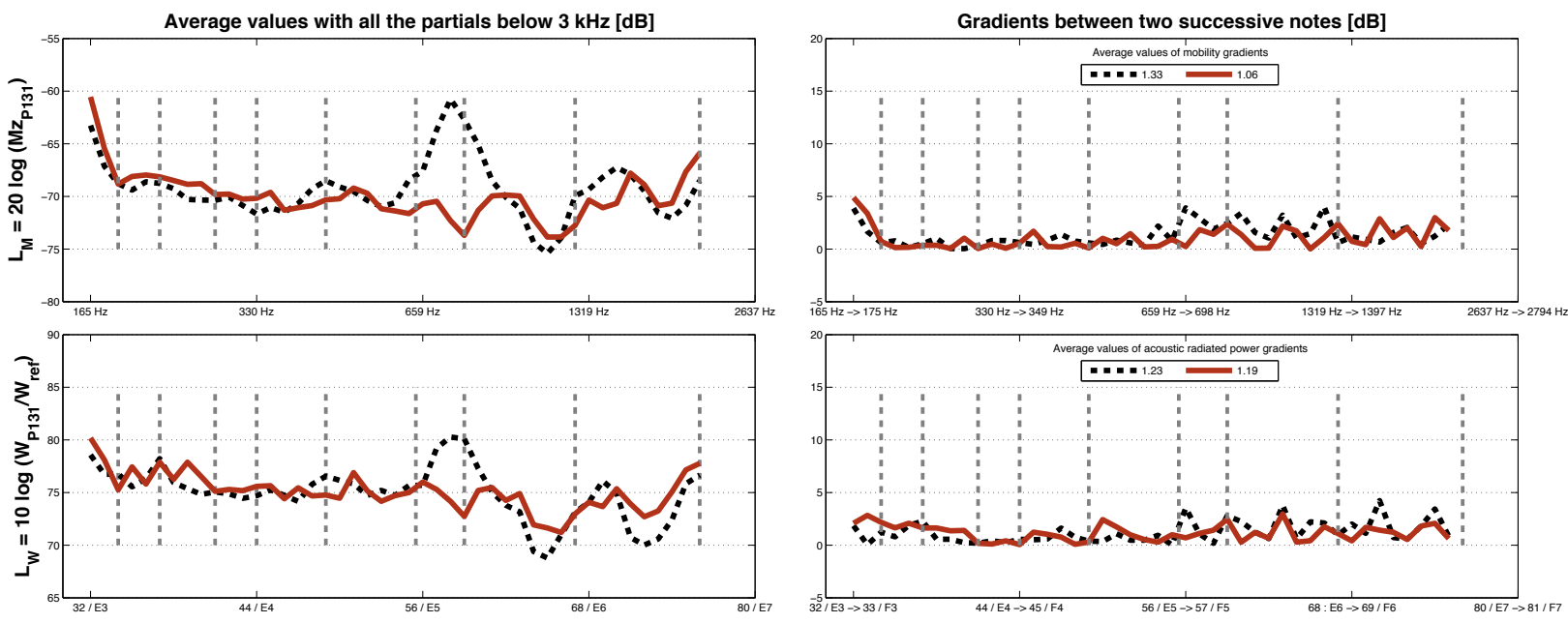

4 Fig. 20 - (Color online) Comparison between the P131 soundboard analyzed in III.C.3 and the P131 soundboard with a continuous main bridge.

6 Concerning the note-to-note gradients, their average values are lower than in the 7 reference case, especially that of mobility (see average values in Fig. 20, right).

8 However, we can see that the gradients of high-pitched notes are lower but still 9 remain high for both indicators. In the case of the acoustic radiated power, it leads to 10 a perceived division or multiplication of this power by two from the previous notes (3 $11 \mathrm{~dB}$ ). For the notes originally placed before the bridge break, few changes can be 12 noted on the mobility gradients. On the contrary, a continuous bridge increases the 13 acoustic radiated power gradients in the frequency band $[165 ; 650] \mathrm{Hz}$. Therefore, as 14 the average radiated power gradients are around the same order of magnitude for 15 both cases, it appears that it is to the detriment of mid-frequency notes (fundamental 
1 frequency lower than $650 \mathrm{~Hz}$ ) and to the advantage of notes with a fundamental 2 frequency higher than $650 \mathrm{~Hz}$.

\section{IV.B. RemOVAL OF RIBS IN THE TREBLE ZONE}

4 Contrary to the previous case, the removal of two ribs has no effect on the first part of 5 the main bridge (below note number 56 in Fig. 21). In this range, the changes are 6 negligible for both indicators, as much for the average values as for the gradients.

7 Obviously, the effect of the main bridge break remains, with a pronounced increase 8 of the two indicators around note number 58 (Fig. 21, left), and also significant local 9 gradients (Fig. 21, right). For high-pitched notes, the two indicators do not follow the 10 same trends, contrary to the previous cases. For high-pitched notes, the mobility

11 level increases because the stiffness becomes lower locally due to the removal of the 12 stiffeners (top left Fig. 21). The loss of mobility from notes 62 to 67 in the reference

13 case (900 to $1250 \mathrm{~Hz}$ ) has now been eliminated. At higher frequencies, mobility 14 increases until note number $71(1550 \mathrm{~Hz})$ and finally decreases. Thus the removal of 15 ribs will lead to a lower sustain due to the quicker injection of string energies in high16 pitched notes.

17 However, this solution presents the advantage of substantially decreasing the 18 gradient between notes: globally the ratio of average gradients is around $3 / 4$ between 19 the two cases (Fig. 21, top right). Focusing above note number 62, this ratio falls to 20 around $1 / 2$ with average gradients of $1.5 \mathrm{~dB}$ and $0.8 \mathrm{~dB}$, respectively, for the 21 reference case and the P131 soundboard without two ribs. 

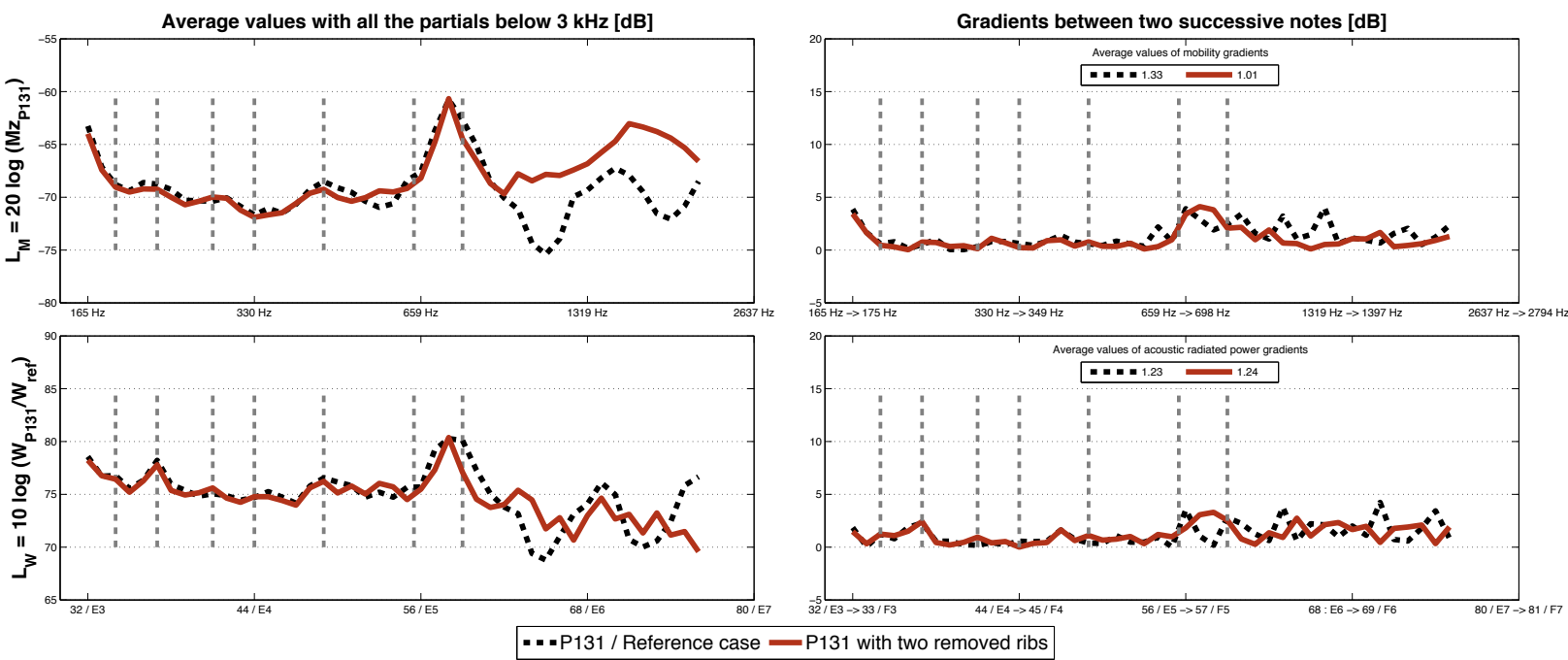

Fig. 21 - (Color online) Comparison between the P131 soundboard analyzed in III.C.3 and the P131 soundboard with ribs 1 and 2 removed.

As for the mobility, the acoustic radiated power is impacted after the bridge break and seems to approximately follow a decreasing linear tendency, passing in the middle of the reference case (Fig. 21, bottom right). However, some variations of radiated power moving to high frequency remain. Consequently, the maximum gradient is only

$9 \quad$ Finally, for the high-pitched notes, removing the ribs implies higher mobility and thus

10 a lower sustain. By way of compensation, the variations of the two indicators are 11 lower than in the reference case. From the musical viewpoint, removing the ribs 12 allows more homogeneous playing with lower variations between consecutive notes 13 for this frequency range.

14 These parametrical studies show the difficulty of controlling the piano soundboard 15 response, particularly in the treble zone, which can be considered as a sensitive 16 range. For these notes, a structural change has a considerable effect on their 
1 behavior, as shown in this article. Moreover, the bridge break homogenizes the

2 behavior of the third and fourth octaves. Regarding the bridge length, it could be

3 interesting to increase it at the beginning without changing the string coupling points,

4 in order to homogenize the behavior of the first notes. We also suggest several

5 improvements in order to reduce global and note-to-note variations in the ranges

$6 \quad$ where the response is inhomogeneous.

7 Considering the design constraints (addition of stiffeners over the whole soundboard

8 due to the stress applied by the strings and breaking the bridge to let the cast iron

9 plate pass through), the reference case demonstrates the skill and knowledge of

10 piano makers. Indeed, their empirical method of design allows them to control the

11 sound produced without scientific prediction tools.

\section{V. Conclusion}

13 This article presented how to model the piano soundboard, that is to say a complex

14 structure with non-rectangular contours, composed of a material with an angle of

15 orthotropy and several stiffeners in positioned in different direction. Here, we limited our

16 study to the case of an upright piano soundboard in the frequency domain $[0 ; 3000] \mathrm{Hz}$.

17 The model developed allowed us to calculate the modes of this complex ribbed

18 structure and classify them into different families: those for which modal shapes are

19 similar to unribbed plate modes, those for which only strong superstructures inhibit the

20 vibration, those for which all superstructures block vibrations, and those for which

21 vibration is localized in different areas of the soundboard. It was shown that these

22 families depend on the frequency ranges and the wavelengths of modes. 
1 Moreover, we also presented maps of the mobility, the space average quadratic velocity

2 and the acoustic radiated power as a function of the excitation points (string coupling

3 positions). Moreover, we showed that focusing on these maps is not fully relevant and

4 introduced indicators that are more accurate from the musical standpoint.

5 We highlighted a frequency range that seems to be difficult to control for piano makers,

6 due to high global and note-to-note variability (high-pitched notes), whereas the low and

7 mid-range frequencies are well mastered by them. Mainly guided by technical

8 constraints, the traditional design appears to be a good solution considering that piano

9 makers do not necessarily use scientific prediction tools.

10 To conclude, although the model presented in this paper suffers from limitations

11 (membrane effects, downbearing and geometrical simplifications), and so does not

12 describe a soundboard in a playing situation, it appears that these limitations do not

13 affect the general trends, meaning that the method is relevant to parametrical studies.

14 Some of the proposals, such as a continuous main bridge and removing ribs for high-

15 pitched notes, provide paths of improvement to make playing more homogenous.

16 However, they imply changing the design of the instrument to make it possible.

\section{Acknowledgments}

18 This work was performed in the framework of the Labex CeLyA of Universite de Lyon,

19 supervised by the French National Research Agency (Nos. ANR-10-LABX-0060/ ANR-

20 11-IDEX-0007).

21 The authors thank Pierre Margerit for the measurements obtained during his Master

22 degree internship at LVA. We are also very grateful to ITEMM (European Technological 
1 Institution for Music Professions) and particularly to Marthe Curtit and Patrick Sinigaglia

2 for having kindly given the Pleyel P131 soundboard used in this study to LVA. 


\section{Appendix A Hamiltonian calculations of a superstructure}

2 In this appendix, we give the main steps of the calculations for expressing the

3 Hamiltonian of an arbitrary superstructure. To make reading simpler, we have chosen a

4 bridge. At the end of the appendix, we give the function for a rib including the change of

5 height.

6 The partial Hamiltonian is defined as:

$$
\mathrm{H}=\int_{t_{0}}^{t_{1}}\left(E_{c}-E_{d}\right) d t
$$

7 Where $E_{c}$ refers to kinetic energy and $E_{d}$ refers to strain energy. $\left[t_{0} ; t_{1}\right]$ is an arbitrary

8 time interval.

9 We first begin by defining the displacement field of the superstructure in direction $\vec{x}$ at 10 position $\mathrm{y}=\mathrm{y}_{\mathrm{c}}$ :

$$
\left\{\begin{array}{l}
u_{c}(x, z, t)=-z w_{, x}\left(x, y_{c}, t\right) \\
v_{c}(x, z, t)=-z w_{, y}\left(x, y_{c}, t\right) \\
w_{c}(x, y, t)=w\left(x, y_{c}, t\right)+\left(y-y_{c}\right) w_{, y}\left(x, y_{c}, t\right)
\end{array}\right.
$$

11 Where $y \in\left[y_{c}-b_{c} / 2 ; y_{c}+b_{c} / 2\right]$ and $z \in\left[h / 2 ; h / 2+H_{c}\right]$ where $h$ and $H$ are 12 respectively the plate thickness and the rib height.

13 The kinetic and strain energies are calculated on the entire bridge volume placed at

$14 \mathrm{y}=\mathrm{y}_{\mathrm{c}}$. The superstructure is defined for $x \in\left[x_{\text {ini }} ; x_{f i n}\right]$. Let the kinetic energy be: 


$$
\mathrm{E}_{\mathrm{c}}=\frac{1}{2} \int_{y=-b_{c} / 2}^{b_{c} / 2} \int_{x=x_{\text {ini }}}^{x_{\mathrm{fin}}=h / 2} \int_{c}^{h / 2+H_{c}} \rho_{c}\left(\dot{u}_{c}^{2}+\dot{v}_{c}^{2}+\dot{w}_{c}^{2}\right) d x d y d z
$$

1 By replacing $\dot{u}_{c}^{2}, \dot{v}_{c}^{2}$ and $\dot{w}_{c}^{2}$ by their expression from A.2 and after making the integrals of 2 variables $y$ and $z$, we obtain:

$$
\mathrm{E}_{\mathrm{c}}=\frac{1}{2} \rho_{c} \int_{x=x_{\text {ini }}}^{x_{\text {fin }}}\left(b_{c} H_{c} \dot{w}^{2}+I_{f c} \dot{w}, x_{, x}^{2}+I_{g c} \dot{w}_{, y}^{2}\right) d x
$$

3 Where the bending inertia is equal to $I_{f c}=\frac{b_{c}}{3}\left[\left(h / 2+H_{c}\right)^{3}-(h / 2)^{3}\right]$ and torsion inertia

4 is $I_{g c}=I_{f c}+\frac{H_{c} b_{c}^{3}}{12}$.

5 For the strain energy, we must calculate the small deformation tensor $\overline{\overline{\boldsymbol{\epsilon}}}=\frac{1}{2}(\operatorname{gr} \overline{\bar{a}} d(\overline{\boldsymbol{U}})+$ $\left.6 \operatorname{gr} \overline{\bar{a}} d(\overline{\boldsymbol{U}})^{t}\right)$ and the strain tensor $\overline{\boldsymbol{\epsilon}}=\overline{\bar{M}}_{s} \overline{\boldsymbol{\sigma}}$ (inverse Hook law). We find the following small 7 deformation tensor in its vector form:

$$
\overline{\boldsymbol{\epsilon}}=\frac{1}{2}\left|\begin{array}{c}
-2 z w_{, x x}\left(x, y_{c}, t\right) \\
0 \\
0 \\
-z w_{, x y}\left(x, y_{c}, t\right) \\
y w_{, x y}\left(x, y_{c}, t\right) \\
0
\end{array}\right|
$$

8 The strains are linked to deformation through a flexibility matrix. The matrix $\overline{\bar{M}}_{S}$ for an 9 orthotropic material is: 


$$
\overline{\bar{M}}_{s}=\left|\begin{array}{ccccc}
\frac{1}{E_{x}} & -\frac{v_{x y}}{E_{x}} & -\frac{v_{x z}}{E_{x}} & & \\
-\frac{v_{y x}}{E_{y}} & \frac{1}{E_{y}} & -\frac{v_{y z}}{E_{y}} & & \\
-\frac{v_{z x}}{E_{z}} & -\frac{v_{z y}}{E_{z}} & \frac{1}{E_{z}} & & \\
& & \frac{1}{2 G_{x y}} & & \\
(0) & & & \frac{1}{2 G_{x z}} & \\
& & & & \frac{1}{2 G_{y z}}
\end{array}\right|
$$

1 Thus, we determine the strain in the bridge:

$$
\left\{\begin{array}{l}
\sigma_{x x}=-z E_{x} w_{, x x}\left(x, y_{c}, t\right) \\
\sigma_{x y}=-z G_{x y} w_{, x y}\left(x, y_{c}, t\right) \\
\sigma_{x z}=y G_{x z} w_{, x y}\left(x, y_{c}, t\right)
\end{array}\right.
$$

2 After which we calculate the strain energy, let:

$$
\mathrm{E}_{\mathrm{d}}=\frac{1}{2} \int_{x=x_{\text {ini }}}^{x_{f i n}}\left(E_{x} I_{f c} w_{, x x}^{2}\left(x, y_{c}, t\right)+D_{g} w_{, x y}^{2}\left(x, y_{c}, t\right)\right) d x
$$

3 With $D_{g}=G_{x y} I_{f c}+G_{x z} \frac{H_{c} b_{c}}{12}$ that we define as the torsion dynamic rigidity of an

4 orthotropic beam in the plane $(\vec{y}, \vec{z})$.

5 For the sake of simplicity, we consider the beam as isotropic in the following. $D_{g}$ 6 becomes $D_{g}=G_{c} I_{g c}$ and we define $E_{c}$ as the Young modulus of the bridge. Thus we 7 express the Hamiltonian of the bridge: 


$$
\begin{aligned}
\mathrm{H}_{\text {bridge }}=\int_{t_{0}}^{t_{1}}\left(\frac { 1 } { 2 } \int _ { x = x _ { \text { in } i } } ^ { x _ { f i n } } \left[\rho_{c}\left(b_{c} H_{c} \dot{w}^{2}\left(x, y_{c}, t\right)+I_{f c} \dot{w}_{, x}^{2}\left(x, y_{c}, t\right)+I_{g c} \dot{w}_{, y}^{2}\left(x, y_{c}, t\right)\right)\right.\right. \\
\left.\left.-\left(E_{c} I_{f c} w_{, x x}^{2}\left(x, y_{c}, t\right)+D_{g} w_{, x y}^{2}\left(x, y_{c}, t\right)\right)\right] d x\right) d t
\end{aligned}
$$

1 That we gather on a line at $\mathrm{y}=\mathrm{y}_{\mathrm{c}}$ :

$$
\begin{aligned}
\mathrm{H}_{\text {bridge }}=\int_{t_{0}}^{t_{1}}\left(\frac { 1 } { 2 } \int _ { x = x _ { \text { ini } } } ^ { x _ { f i n } } \int _ { y = 0 } ^ { L _ { y } } \left[\rho_{c}\left(b_{c} H_{c} \dot{w}^{2}(x, y, t)+I_{f c} \dot{w_{, x}^{2}}(x, y, t)+I_{g c} \dot{w}_{, y}^{2}(x, y, t)\right)\right.\right. \\
\left.\left.-\left(E_{c} I_{f c} w_{, x x}^{2}(x, y, t)+G_{c} I_{g c} w_{, x y}^{2}(x, y, t)\right)\right] \delta\left(y-y_{c}\right) d x d y\right) d t
\end{aligned}
$$

2 The same approach is followed for the ribs. Beyond the change of orientation, the only 3 change is that the height of the ribs is different at the extremities (around $5 \mathrm{~mm}$ ). See

4 Fig. 7-b. Thus the integral along the direction of the rib can be split: the two extremities

5 with a height of $5 \mathrm{~mm}$ and the middle part with a height of $\mathrm{H}$. Finally, we consider a

6 number of rib $\mathrm{N}_{\mathrm{r}}$ equal to the sum of ribs and extremities. Thus we give the expression 7 of the Hamiltonian of rib:

$$
\begin{aligned}
\mathrm{H}_{\mathrm{rib}}=\int_{t_{0}}^{t_{1}}\left(\frac { 1 } { 2 } \int _ { x = 0 } ^ { L _ { x } } \int _ { y = y _ { i n i } } ^ { y _ { f i n } } \left[\rho_{r}\left(b H \dot{w}^{2}(x, y, t)+I_{f} \dot{w}_{, y}^{2}(x, y, t)+I_{g} \dot{w}_{, x}^{2}(x, y, t)\right)\right.\right. \\
\left.\left.\quad-\left(E_{r} I_{f} w_{, y y}^{2}(x, y, t)+G_{r} I_{g} w_{, x y}^{2}(x, y, t)\right)\right] \delta\left(x-x_{r}\right) d x d y\right) d t
\end{aligned}
$$

8 


\section{Bibliography}

2 [1] I. Brémaud, "Acoustical properties of wood in string instruments soundboards 3 and tuned idiophones: Biological and cultural diversity," J. Acoust. Soc. Am., vol. 131, 4 no. 1, pp. 807-818, 2012.

5 [2] H. Fletcher, "Normal vibration frequencies of a stiff piano string," J. Acoust. Soc. $6 \quad$ Am., vol. 36, no. 1, pp. 203-209, 1964.

7 [3] G. Weinreich, "Coupled piano strings," J. Acoust. Soc. Am., vol. 62, no. 6, pp. 8 1474-1484, 1977.

9 [4] J. Kindel and I. C. Wang, "Vibrations of a piano soundboard: Modal analysis and 10 finite element analysis.," J. Acoust. Soc. Am., vol. 81, pp. 61-61, 1987.

11 [5] H. Suzuki, "Vibration and sound radiation of a piano soundboard," J. Acoust. Soc. 12 Am., vol. 80, no. 6, pp. 1573-1582, 1986.

13 [6] H. Suzuki and I. Nakamura, "Acoustics of Pianos," Appl. Acoust., vol. 30, pp. 14 147-205, 1990.

15 [7] H. A. Conklin Jr, "Design and tone in the mechanoacoustic piano. Part I. Piano 16 hammers and tonal effects," J. Acoust. Soc. Am., vol. 99, no. 6, pp. 3286-3296, 1996.

17 [8] H. A. Conklin Jr, "Design and tone in the mechanoacoustic piano.Part II. Piano 18 structure," J. Acoust. Soc. Am., vol. 100, no. 2, pp. 695-708, 1996.

19 [9] H. A. Conklin Jr, "Design and tone in the mechanoacoustic piano. Part III. Piano 20 strings and scale design," J. Acoust. Soc. Am., vol. 100, no. 3, pp. 1286-1298, 1996.

21 [10] N. Giordano, "Simple model of a piano soundboard," J. Acoust. Soc. Am., vol. 22 102, no. 2, pp. 1159-1168, 1997.

23 [11] N. Giordano, "Mechanical impedance of a piano soundboard," J. Acoust. Soc.

24 Am., vol. 103, no. 4, pp. 2128-2133, 1998.

25 [12] N. Giordano, "Sound production by a vibrating piano soundboard: Experiment," 26 J.-Acoust. Soc. Am., vol. 104, no. 3, pp. 1648-1653, 1998.

27 [13] P. Dérogis and R. Caussé, "Computation and modelisation of the sound radiation 28 of an upright piano using modal formalism and integral equations," in ICA: International 29 Congress on Acoustic, 1995, vol. 3, pp. 409-412. 
1 [14] J. Berthaut, M. N. Ichchou, and L. Jézéquel, "Piano soundboard: structural

2 behavior, numerical and experimental study in the modal range," Appl. Acoust., vol. 64, 3 no. 11, pp. 1113-1136, Nov. 2003.

4 [15] J. Bensa, S. Bilbao, R. Kronland-Martinet, and J. O. Smith, "The simulation of 5 piano string vibration: From physical models to finite difference schemes and digital 6 waveguides," J. Acoust. Soc. Am., vol. 114, no. 2, p. 1095, 2003.

7 [16] A. Stulov, "Physical modelling of the piano string scale," Appl. Acoust., vol. 69, 8 no. 11, pp. 977-984, Nov. 2008.

9 [17] K. Ege, X. Boutillon, and M. Rébillat, "Vibroacoustics of the piano 10 soundboard:(Non) linearity and modal properties in the low-and mid-frequency ranges,"

11 J. Sound Vib., vol. 332, no. 5, pp. 1288-1305, 2013.

12 [18] X. Boutillon and K. Ege, "Vibroacoustics of the piano soundboard: Reduced 13 models, mobility synthesis, and acoustical radiation regime," J. Sound Vib., vol. 332, no.

14 18, pp. 4261-4279, Sep. 2013.

15 [19] A. Chaigne, B. Cotté, and R. Viggiano, "Dynamical properties of piano 16 soundboards," J. Acoust. Soc. Am., vol. 133, no. 4, pp. 2456-2466, 2013.

17 [20] J. Chabassier, A. Chaigne, and P. Joly, "Modeling and simulation of a grand 18 piano," J. Acoust. Soc. Am., vol. 134, no. 1, pp. 648-665, 2013.

19 [21] F. Rigaud, "Models of music signals informed by physics. Application to piano 20 music analysis by non-negative matrix factorization.," Télécom ParisTech, 2013.

21 [22] N. Etchenique, S. R. Collin, and T. R. Moore, "Coupling of transverse and 22 longitudinal waves in piano strings," J. Acoust. Soc. Am., vol. 137, no. 4, pp. 1766$231771,2015$.

24 [23] P. Guillaume, "Device for producing signals representative of sounds of a 25 keyboard and stringed instrument," US20090241757 A1, 01-Oct-2009.

26 [24] J.-L. Guyader, Vibration in Continuous Media. London: ISTE Ltd., 2006.

27 [25] A. W. Leissa, Vibration of plates, vol. 160. Scientific and Technical Information

28 Division, National Aeronautics and Space Administration, 1969.

29 [26] B. Laulagnet, "Sound radiation by a simply supported unbaffled plate," J. Acoust. 30 Soc. Am., vol. 103, no. 5, pp. 2451-2462, 1998. 
1 [27] D. J. Mead and A. K. Mallik, "An approximate method of predicting the response

2 of periodically supported beams subjected to random convected loading," J. Sound Vib., 3 vol. 47, no. 4, pp. 457-471, 1976.

4 [28] R. D. Mindlin, "Influence of rotary inertia and shear on flexural motion of isotropic

5 elastic plate," J. Appl. Mech.-Trans. Asme, vol. 18, no. 1, pp. 31-38, 1951.

6 [29] C. Lesueur, Acoustic radiation of structures: vibroacoustic and fluid / structure 7 interactions. Eyrolles, 1988.

8 [30] P. R. Stepanishen, "Modal coupling in the vibration of fluid-loaded cylindrical 9 shells," J. Acoust. Soc. Am., vol. 71, no. 4, pp. 813-823, Apr. 1982.

10 [31] C. E. Wallace, "Radiation Resistance of a Rectangular Panel," J. Acoust. Soc. 11 Am., vol. 51, no. 3, pp. 946-952, 1972.

12 [32] A. Putra and D. J. Thompson, "Sound radiation from rectangular baffled and 13 unbaffled plates," Appl. Acoust., vol. 71, no. 12, pp. 1113-1125, Dec. 2010.

14 [33] C. Wang, "Modal sound transmission loss of a single leaf panel: Effects of inter15 modal coupling," J. Acoust. Soc. Am., vol. 137, no. 6, pp. 3514-3522, 2015.

16 [34] G. Maidanik, "Response of Ribbed Panels to Reverberant Acoustic Fields," J.

17 Acoust. Soc. Am., vol. 34, no. 6, pp. 809-826, 1962.

18 [35] G.-F. Lin and J. M. Garrelick, "Sound transmission through periodically framed 19 parallel plates," J. Acoust. Soc. Am., vol. 61, no. 4, pp. 1014-1018, Apr. 1977.

20 [36] D. J. Mead and S. Parthan, "Free wave propagation in two-dimensional periodic 21 plates," J. Sound Vib., vol. 64, no. 3, pp. 325-348, Jun. 1979.

22 [37] R. Vaicaitis and M. Slazak, "Noise transmission through stiffened panels," J.

23 Sound Vib., vol. 70, no. 3, pp. 413-426, Jun. 1980.

24 [38] B. R. Mace, "Periodically stiffened fluid-loaded plates, I: Response to convected 25 harmonic pressure and free wave propagation," J. Sound Vib., vol. 73, no. 4, pp. 47326 486, Dec. 1980.

27 [39] B. R. Mace, "Periodically stiffened fluid-loaded plates, II: Response to line and 28 point forces," J. Sound Vib., vol. 73, no. 4, pp. 487-504, Dec. 1980.

29 [40] B. R. Mace, "Sound radiation from a plate reinforced by two sets of parallel 30 stiffeners," J. Sound Vib., vol. 71, no. 3, pp. 435-441, Aug. 1980. 
1 [41] A. Mejdi and N. Atalla, "Dynamic and acoustic response of bidirectionally

2 stiffened plates with eccentric stiffeners subject to airborne and structure-borne

3 excitations," J. Sound Vib., vol. 329, no. 21, pp. 4422-4439, Oct. 2010.

4 [42] J. Legault, A. Mejdi, and N. Atalla, "Vibro-acoustic response of orthogonally

5 stiffened panels: The effects of finite dimensions," J. Sound Vib., vol. 330, no. 24, pp.

6 5928-5948, Nov. 2011.

7 [43] J. Legault and N. Atalla, "Sound transmission through a double panel structure

8 periodically coupled with vibration insulators," J. Sound Vib., vol. 329, no. 15, pp. 3082-

9 3100, Jul. 2010.

10 [44] F. X. Xin, "An exact elasticity model for rib-stiffened plates covered by decoupling

11 acoustic coating layers," Compos. Struct., vol. 119, pp. 559-567, Jan. 2015.

12 [45] M. T. Chang and R. Vaicaitis, "Noise transmission into semicylindrical enclosures

13 through discretely stiffened curved panels," J. Sound Vib., vol. 85, no. 1, pp. 71-83,

14 Nov. 1982.

15 [46] J.-L. Guyader and B. Laulagnet, "Sound radiation by finite cylindrical ring

16 stiffened shells," J. Sound Vib., vol. 138, no. 2, pp. 173-191, 1990.

17 [47] D. E. Beskos and J. B. Oates, "Dynamic analysis of ring-stiffened circular

18 cylindrical shells," J. Sound Vib., vol. 75, no. 1, pp. 1-15, Mar. 1981.

19 [48] L. R. Koval, "On sound transmission into a stiffened cylindrical shell with rings

20 and stringers treated as discrete elements," J. Sound Vib., vol. 71, pp. 511-521, Aug.

211980.

22 [49] D. J. Mead and N. S. Bardell, "Free vibration of a thin cylindrical shell with

23 discrete axial stiffeners," J. Sound Vib., vol. 111, no. 2, pp. 229-250, Dec. 1986.

24 [50] D. J. Mead and N. S. Bardell, "Free vibration of a thin cylindrical shell with

25 periodic circumferential stiffeners," J. Sound Vib., vol. 115, no. 3, pp. 499-520, 1987.

26 [51] C. H. Hodges, J. Power, and J. Woodhouse, "The low frequency vibration of a

27 ribbed cylinder, Part 1: Theory," J. Sound Vib., vol. 101, no. 2, pp. 219-235, 1985.

28 [52] C. H. Hodges, J. Power, and J. Woodhouse, "The low frequency vibration of a

29 ribbed cylinder, Part 2: Observations and interpretation," J. Sound Vib., vol. 101, no. 2,

30 pp. 237-256, Jul. 1985. 
1 [53] C. B. Burroughs, "Acoustic radiation from fluid-loaded infinite circular cylinders

2 with doubly periodic ring supports," J. Acoust. Soc. Am., vol. 75, no. 3, pp. 715-722, 3 Mar. 1984.

4 [54] A. Mejdi and N. Atalla, "Vibroacoustic analysis of laminated composite panels 5 stiffened by complex laminated composite stiffeners," Int. J. Mech. Sci., vol. 58, no. 1, 6 pp. 13-26, May 2012.

7 [55] A. Mejdi, J. Legault, and N. Atalla, "Transmission loss of periodically stiffened 8 laminate composite panels: Shear deformation and in-plane interaction effects," $J$. 9 Acoust. Soc. Am., vol. 131, no. 1, pp. 174-185, 2012.

10 [56] F. X. Xin and T. J. Lu, "Analytical modeling of fluid loaded orthogonally rib-

11 stiffened sandwich structures: Sound transmission," J. Mech. Phys. Solids, vol. 58, no.

12 9, pp. 1374-1396, Sep. 2010.

13 [57] F. X. Xin and T. J. Lu, "Analytical modeling of wave propagation in orthogonally 14 rib-stiffened sandwich structures: Sound radiation," Comput. Struct., vol. 89, no. 5-6, 15 pp. 507-516, Mar. 2011.

16 [58] F. X. Xin and T. J. Lu, "Sound radiation of orthogonally rib-stiffened sandwich 17 structures with cavity absorption," Compos. Sci. Technol., vol. 70, no. 15, pp. 219818 2206, Dec. 2010.

19 [59] F. X. Xin and T. J. Lu, "Transmission loss of orthogonally rib-stiffened double20 panel structures with cavity absorption," J. Acoust. Soc. Am., vol. 129, no. 4, pp. 191921 1934, 2011.

22 [60] B. Trévisan, K. Ege, and B. Laulagnet, "Vibroacoustics of orthotropic plates 23 ribbed in both directions: Application to stiffened rectangular wood panels," J. Acoust.

24 Soc. Am., vol. 139, no. 1, pp. 227-246, Jan. 2016.

25 [61] C. Waltham and A. Kotlicki, "Vibrational characteristics of harp soundboards," J. 26 Acoust. Soc. Am., vol. 124, no. 3, p. 1774, 2008.

27 [62] J.-L. Le Carrou, Q. Leclere, and F. Gautier, "Some characteristics of the concert 28 harp's acoustic radiation," J. Acoust. Soc. Am., vol. 127, no. 5, p. 3203, 2010.

29 [63] J.-L. Guyader and B. Laulagnet, "Structural acoustic radiation prediction:

30 Expending the vibratory response on a functional basis," Appl. Acoust., vol. 43, no. 3 , 
1 pp. 247-269, 1994.

2 [64] M. Géradin and D. Rixen, Theory of vibrations: dynamic of structures. Masson, 31996.

4 [65] A. Chaigne, J. Kergomard, X. Boutillon, J.-P. Dalmont, and Collectif, Acoustic of 5 musical instruments. Paris: Belin, 2008.

6 [66] K. Ege, "The piano soundboard: Modal studies in low and mid frequencies.," 7 Ecole Polytechnique X, 2009.

8 [67] K. Ege, "La table d'harmonie du piano-Études modales en basses et moyennes 9 fréquences," Ecole Polytechnique X, 2009.

10 [68] C. E. Wallace, "The acoustic radiation damping of the modes of a rectangular 11 panel," J. Acoust. Soc. Am., vol. 81, no. 6, pp. 1787-1794, Jun. 1987.

12 [69] B. Laulagnet and J.-L. Guyader, "C-valor: benchmarks for vibroacoustic 13 software.," Société française des mécaniciens, Courbevoie (France), pp. 1-118, 1996.

14 [70] A. Berry and J. Nicolas, "Vibration and sound radiation of complex plates: A 15 variational approach.," J. Acoust. Soc. Am., vol. 90, no. 4, pp. 2249-2250, Oct. 1991.

16 [71] R. F. Keltie and H. Peng, "The Effects of Modal Coupling on the Acoustic Power 17 Radiation From Panels," J. Vib. Acoust. Stress Reliab. Des., vol. 109, no. 1, pp. 48-54, 18 Jan. 1987.

19 [72] D. Fandrich, "Soundboard Technology \& Manufacture," presented at the 20 PianoTalk \#2, 1995.

21 [73] P. Margerit, B. Trévisan, K. Ege, and B. Laulagnet, "Vibroacoustic behavior of an 22 upright piano soundboard: comparison between experiment and analytical modelling.," 23 presented at the 22ème Congrès Français de Mécanique, Lyon, 2015.

24 [74] T. Moore and S. Zietlow, "Interferometric studies of a piano soundboard," Stud.25 Fac. Collab. Res., Mar. 2006.

26 [75] K. Wogram, "Acoustical research on pianos. Part I: Vibrational characteristics of 27 the sound- board.," Musikinstrum., vol. 24, pp. 694-702, 1980.

28 [76] P. Dérogis and R. Caussé, "Characterization of the acoustic radiation of the 29 soundboard of an upright piano.," J. Phys., vol. 4, no. C5, pp. 609-612, 1994.

30 [77] I. Nakamura, "The vibrational character of the piano soundboard.," Proc. 11th 
1 ICA, vol. 4, pp. 385-388, 1983.

2 [78] I. Elishakoff, "Distribution of natural frequencies in certain structural elements," $J$.

3 Acoust. Soc. Am., vol. 57, no. 2, pp. 361-369, Feb. 1975.

4 [79] I. J. Busch-Vishniac, "Drive point impedance of an infinite orthotropic plate under

5 tension," J. Acoust. Soc. Am., vol. 71, no. 2, p. 368, 1982.

6 [80] A. Mamou-Mani, J. Frelat, and C. Besnainou, "Numerical simulation of a piano

7 soundboard under downbearing," J. Acoust. Soc. Am., vol. 123, no. 4, pp. 2401-2406,

8 Apr. 2008. 
Fig. 1 - (Color online) Upright piano soundboard of Pleyel P131 with $E_{x}$ and $E_{y}$ being the strong and the weak Young's moduli of the wood. View from front side (bridge side) with ribs and cut-off corner beams added in transparency.

Fig. 2 - (Color online) The addition of springs on a rectangular simply supported plate. On the left: the initial plate with specific orthotropy; on the right: the addition of springs. The non-rectangular contours and the non-specified angle of orthotropy are well described.

Fig. 3 - (Color online) Examples of possible geometries with different orthotropy angles and edges.

Fig. 4 - (Color online) Influence of spring rigidity. a) Average quadratic velocity ratio for two spring rigidities. Solid line: sufficient rigidity; dashed line: insufficient rigidity. The excitation is placed at $\left(\mathrm{x}_{\mathrm{e}}, \mathrm{y}_{\mathrm{e}}\right)=(1.26,0.76)$. b) Modal density per octave for two spring rigidities and analytical plot from [11], [17], [67] Eq. (A.13): circle markers: sufficient spring rigidity; square markers: insufficient spring rigidity; solid line: analytical expression.

Fig. 5 - (Color online) Modal shapes of a clamped non-rectangular plate with an angle of orthotropy of $34.8^{\circ}$. Left: insufficient spring rigidity; Center: sufficient spring rigidity; Right: FEM-NASTRAN with a mesh of $5 \mathrm{~mm}$.

Fig. 6 - (Color online) Geometry modelled for the Pleyel P131.

Fig. 7 - (Color online) Rib geometry. a) Section view of a real soundboard rib. b) Simplified rib in the present model. c) Partial front view of the soundboard.

Fig. 8 - (Color online) Classification of the first 200 modes of a Pleyel P131 soundboard.

Fig. 9 - (Color online) Low frequency modes similar to unribbed plate modes.

Fig. 10 - (Color online) Modes where the transverse displacement is strongly minimized by the bridges.

Fig. 11 - (Color online) Modes where the transverse displacement is strongly minimized by all the superstructures.

Fig. 12 - (Color online) Modes where the vibrations are localized into areas delimited by superstructures.

Fig. 13 - (Color online) Evolution of local and general indicators at string/main bridge coupling points: Top: mobility; Center: space average quadratic velocity; Bottom: Acoustic radiated power.

Fig. 14 - (Color online) Evolution of mobility (top) and acoustic radiated power (bottom) of a P131 soundboard. Left: dB values of indicators; Right: gradients (absolute values) between two successive notes in $\mathrm{dB}$.

35

36

37

38

39

40

Fig. 15 - (Color online) Comparison of Pleyel P131 modal shapes between our analytical modelling and experimental results.

Fig. 16 - (Color online) Nastran FEM model of Pleyel P131 soundboard with the same geometrical simplifications as in our model. a) and c) view of ribs view with cut-off corner beams; b) and d) bridge view. 
Fig. 17 - (Color online) Eigen-frequency convergences for our model and the Nastran FEM model (without membrane) as a function of truncation order and the mesh size.

Fig. 18 - (Color online) Comparison of numerical and experimental modal shapes using four methods. From left to right: analytical modelling, Nastran FEM without membrane, Nastran FEM with membrane and experimental results.

Fig. 19 - (Color online) Different sets of upright piano soundboards based on the Pleyel P131 configuration. a) Reference case: Pleyel P131; b) Continuous main bridge; c) Removal of ribs for high-pitched notes.

Fig. 20 - (Color online) Comparison between the P131 soundboard analyzed in III.C.3 and the P131 soundboard with a continuous main bridge.

Fig. 21 - (Color online) Comparison between the P131 soundboard analyzed in III.C.3 and the P131 soundboard with ribs 1 and 2 removed. 


\begin{tabular}{c|c}
\hline \hline$\rho\left[\mathrm{kg} \cdot \mathrm{m}^{-3}\right]$ & 440 \\
\hline $\mathrm{h}[\mathrm{m}]$ & 0,007 \\
\hline $\mathrm{L}_{\mathrm{x}}[\mathrm{m}]$ & 1,61 \\
\hline $\mathrm{L}_{\mathrm{y}}[\mathrm{m}]$ & 1,56 \\
\hline $\mathrm{E}_{\mathrm{x}}[\mathrm{Pa}]$ & $1,59 \mathrm{E}^{10}$ \\
\hline $\mathrm{E}_{\mathrm{y}}[\mathrm{Pa}]$ & $6,90 \mathrm{E}^{8}$ \\
\hline $\mathrm{nu}$ & 0,44 \\
\hline $\mathrm{nu}$ & 0, \\
\hdashline $\mathrm{G}_{\mathrm{xy}}[\mathrm{Pa}]$ & $0,20 \mathrm{E}+08$ \\
\hline \hline
\end{tabular}

Table 1 - Properties of the extended orthotropic spruce plate.

2

\begin{tabular}{c|c|c|c|c|c}
\hline \hline & $\begin{array}{c}\text { Main bridge } \\
\text { part 1 }\end{array}$ & $\begin{array}{c}\text { Main bridge } \\
\text { part 2 }\end{array}$ & $\begin{array}{c}\text { Bass } \\
\text { bridge }\end{array}$ & $\begin{array}{c}\text { Cut-off corner } \\
\text { beam 1 }\end{array}$ & $\begin{array}{c}\text { Cut-off corner } \\
\text { beam 2 }\end{array}$ \\
\hline$\rho_{c}\left[\mathrm{~kg} \cdot \mathrm{m}^{-3}\right]$ & & & 674 & & \\
\hline $\mathrm{H}_{\mathrm{c}}[\mathrm{m}]$ & & 0.033 & 0.038 & 0.0185 & 0.0192 \\
\hline $\mathrm{b}_{\mathrm{c}}[\mathrm{m}]$ & 0.035 & 0.0325 & & \\
\hline $\mathrm{E}_{\mathrm{c}}[\mathrm{Pa}]$ & & $1.40 \mathrm{E}+10$ & \\
\hline $\mathrm{G}_{\mathrm{c}}[\mathrm{Pa}]$ & & $4.70 \mathrm{E}+008$ & \\
\hline $\mathrm{y}_{\mathrm{c}}[\mathrm{m}]$ & 0.78 & 0.72 & 0.48 & 0.26 & 1.27 \\
\hline $\mathrm{L}[\mathrm{m}]$ & 0.88 & 0.41 & 0.47 & 0.51 & 0.52 \\
\hline \hline
\end{tabular}

Table 2 - Dimensions and properties of bridges and "cut-off" corner beams. 


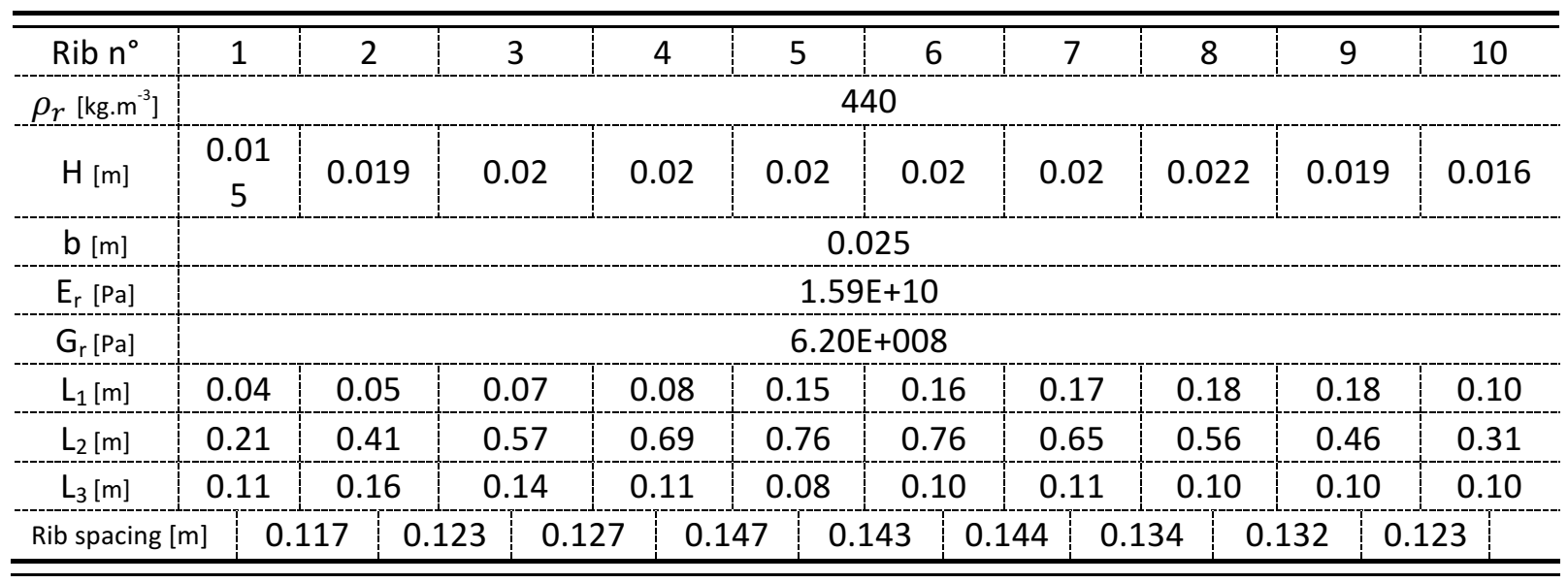

Table 3 - Dimensions and properties of ribs.

\begin{tabular}{|c|c|c|c|}
\hline $\mathrm{L}_{\mathrm{x}}$ & Plate length & $\mathrm{L}_{\mathrm{y}}$ & Plate width \\
\hline$\rho$ & Plate mass density & $\mathrm{h}$ & Plate thickness \\
\hline $\mathrm{D}_{\mathrm{i}}$ & Plate dynamic stiffness & $\rho_{r}$ & Rib mass density \\
\hline $\mathrm{E}_{\mathrm{r}}$ & Rib Young's modulus & $\mathrm{H}$ & Rib height \\
\hline $\mathrm{b}$ & Rib width & $I_{f}$ & Rib momentum of bending inertia \\
\hline $\mathrm{G}_{\mathrm{r}}$ & Rib shear modulus & $\mathrm{I}_{\mathrm{g}}$ & Rib momentum of torsion inertia \\
\hline $\mathrm{E}_{\mathrm{c}}$ & Bridge Young's modulus & $\mathrm{H}_{\mathrm{c}}$ & Bridge height \\
\hline$b_{c}$ & Bridge width & $\mathrm{I}_{\mathrm{fc}}$ & $\begin{array}{l}\text { Bridge momentum of bending } \\
\text { inertia }\end{array}$ \\
\hline $\mathrm{G}_{\mathrm{c}}$ & Bridge shear modulus & $\mathrm{I}_{\mathrm{gc}}$ & $\begin{array}{l}\text { Bridge momentum of torsion } \\
\text { inertia }\end{array}$ \\
\hline$\rho_{c}$ & Bridge mass density & $\mathrm{H}_{\text {plate }}$ & Plate Hamiltonian \\
\hline $\mathrm{H}_{\mathrm{rib}}$ & Rib Hamiltonian & $\mathrm{H}_{\text {bridge }}$ & Bridge Hamiltonian \\
\hline
\end{tabular}

